\title{
Compléments au volume 1 des Hyménoptères Sphecidae d'Europe occidentale (Faune de France 79)
}

Jacques Bitsch, Jean Leclercq

\begin{abstract}
Complements to volume I of the Hymenoptera Sphecidae from Western Europe (Faune de France 79). The present additional material intends to update vol. 79 of the " Faune de France" of Sphecidae (currently referred to as apoid wasps) which was published in 1993. The added material reports new points of view on the phylogeny and classification of these Hymenoptera. It also provides data on the taxonomy and geographical distribution of the different species of Crabroninae (tribes Crabronini and Oxybelini) known from the Western European fauna. Data are based on original observations and on literature (more than 300 bibliographic references).
\end{abstract}

\section{Résumé}

Ces "Compléments" sont destinés à actualiser le volume 79 de la Faune de France des Hyménoptères Sphecidae, paru en 1993. Le texte fournit une mise à jour de la phylogénie et de la classification de ces Insectes (actuellement désignés sous le nom d'Apoïdes Sphéciformes) et un ensemble d"informations nouvelles sur la taxonomie et la répartition des différentes espèces de Crabroninae (tribus des Crabronini et Oxybelini) de la faune d'Europe occidentale. Ces informations sont basées sur des observations originales et sur les données tirées de la littérature (plus de 300 références).

\section{Citer ce document / Cite this document :}

Bitsch Jacques, Leclercq Jean. Compléments au volume 1 des Hyménoptères Sphecidae d'Europe occidentale (Faune de France 79). In: Bulletin de la Société entomologique de France, volume 114 (2),2009. pp. 211-244;

https://www.persee.fr/doc/bsef_0037-928x_2009_num_114_2_2814

\section{Ressources associées :}

Sphecidae

Fichier pdf généré le 08/10/2019 


\title{
Compléments au volume 1 des Hyménoptères Sphecidae d'Europe occidentale (Faune de France 79)
}

\author{
par Jacques BITSCH* et Jean LECLERCQ** \\ * 30 rue du lac d'Oô, F - 31500 Toulouse<colette.bitsch@orange.fr> \\ ** 190 rue Bois-de-Breux, B - 4020 Liège-Jupille, Belgique
}

\begin{abstract}
Résumé. - Ces "Compléments" sont destinés à actualiser le volume 79 de la Faune de France des Hyménoptères Sphecidae, paru en 1993. Le texte fournit une mise à jour de la phylogénie et de la classification de ces Insectes (actuellement désignés sous le nom d'Apoïdes Sphéciformes) et un ensemble d'informations nouvelles sur la taxonomie et la répartition des différentes espèces de Crabroninae (tribus des Crabronini et Oxybelini) de la faune d'Europe occidentale. Ces informations sont basées sur des observations originales et sur les données tirées de la littérature (plus de 300 références).
\end{abstract}

Summary. - Complements to volume I of the Hymenoptera Sphecidae from Western Europe (Faune de France 79). The present additional material intends to update vol. 79 of the "Faune de France" of Sphecidae (currently referred to as apoid wasps) which was published in 1993. The added material reports new points of view on the phylogeny and classification of these Hymenoptera. It also provides data on the taxonomy and geographical distribution of the different species of Crabroninae (tribes Crabronini and Oxybelini) known from the Western European fauna. Data are based on original observations and on literature (more than 300 bibliographic references).

Keywords. - Apoid wasps, phylogeny, classification, geographical repartition, Western Europe.

Le premier volume d'une série de trois ouvrages consacrés aux Hyménoptères Sphecidae d'Europe occidentale, a paru en 1993. Ce volume 1 (BITSCH \& LECLERCQ, 1993) comportait des généralités sur les Sphecidae et une étude détaillée des espèces groupées dans la sousfamille des Crabroninae (Crabronini et Oxybelini). Depuis cette date, de nombreux articles ont apporté des conceptions nouvelles sur la phylogénie et la classification des Sphecidae (s. l.), groupe désigné actuellement sous le nom d'Apoïdes Sphéciformes, et ont fourni un ensemble important de données nouvelles sur la taxonomie et la répartition géographique des espèces, rendant nécessaire la mise à jour des connaissances. Le texte présenté ici, sous forme de compléments et rectifications au volume I, suit l'ordre adopté précédemment en renvoyant aux diverses pages du volume. Il utilise à la fois les données de la littérature, jusqu'à la fin de l'année 2008, et des données originales fournies par des collègues et nos propres récoltes. Chaque fois que cela a été possible, les informations ont été étendues à l'ensemble de l'Europe, tout en privilégiant le territoire français et les pays avoisinants.

La réalisation des présents "Compléments" n'a été possible qu'avec l'aide de nombreux entomologistes, qui ont bien voulu nous fournir des remarques sur le texte initial et des informations nouvelles. Nous remercions en particulier Jan Smit pour les inventaires détaillés de ses récoltes d'Hyménoptères faites en France au cours de ces dernières années, ainsi que tous les entomologistes qui nous ont communiqué leurs récoltes récentes.

p. 10, ligne 7. Lire : preomaulal area [non preomaular area].

p. 14, tableau 1, nervures. Lire : transverso-cubitales [non transverso-cuvitales].

p. 20. ClÉ DES FAMILLES D'ACULÉATES PRÉSENTES EN EUROPE.

$\S 1$. Nous avons utilisé dans cette clé le terme de Formicidae pour désigner le groupe, parfois nommé Formicoidea, comprenant diverses sous-familles, en particulier les Formicinae, 
Myrmecinae, Ponerinae, etc. Cette nomenclature est notamment celle retenue dans le catalogue de Bolton (1995) et dans l'analyse phylogénétique de Brothers (1999). Par ailleurs, les termes d'Apidae et Sphecidae employés dans cette clé correspondent au sens ancien de ces mots (voir ci-dessous : Phylogénie et classification).

p. 21. A la suite d'une confusion de dessins, la légende de la fig. 10a doit être modifiée. Le nom de la fourmi représentée est Pachycondyla (= Mesoponera) castanea [et non Solenopsis invicta].

\section{p. 22, § 12. Lire : Lobes mésosternaux... (au lieu de "Lobes métasternaux...").}

p. 24, tableau 3, liste des genres. Lire : Prionyx Vander Linden [non Wander Linden]. Les crochets entourant le nom du genre Polemistus doivent être supprimés, car le genre est maintenant connu de France. Le mot de Larrinae doit être abaissé d'une ligne, pour se trouver placé au même niveau que la tribu des Larrini (supprimer l'italique) et le genre Larra (à mettre en italique). Dans ce tableau, les Dinetini apparaissent inclus dans les Larrinae, alors qu'ils sont à placer parmi les Astatinae.

p. 25. Au milieu du tableau 3, lire Mellinini [au lieu de Mellini]. Dans ce tableau, les Mellinini ont été placés parmi les Nyssoninae, conformément à la classification de BOHART \& MENKE (1976). Mais des analyses cladistiques ultérieures, notamment celles d'ALEXANDER (1992b) et de PRENTICE (1998), ont montré que les Mellinini forment un groupe (élevé au rang de sous-famille, les Mellininae) nettement séparé des Bembicinae, mais rapproché des Dinetinae (voir ci-dessous). C'est cette seconde conception qui a déjà été retenue dans la rédaction originale du vol. 2 de la présente Faune (p. 107).

p. 26 à 30. PhylogÉNIE ET ClASSIFICATION DES ApOÏDES SPHÉCIFORMES (ANCIENS SPHECIDAE S. L.)

Dans le volume I de la présente Faune, nous avons donné un résumé des principales conceptions présentées par les auteurs concernant les relations phylogénétiques entre les différentes lignées d'Hyménoptères aculéates, en tenant compte des premières analyses cladistiques réalisées sur ces insectes, principalement sur la base de leurs caractéristiques morphologiques. Des travaux ultérieurs, en particulier ceux de MELO (1999) et de BROTHERS (1999), ont confirmé la plupart des conclusions déjà entrevues, tout en les modifiant sur certains points. Nous avons rendu compte brièvement de ces dernières analyses dans le volume 2 de la Faune (BITSCH et al., 1997) et dans l'Avant-propos du vol. 3 (BITSCH et al., 2001).

Il convient aussi de tenir compte de l'étude détaillée de PRENTICE (1998), thèse non publiée dont nous n'avions pas eu connaissance auparavant. Le travail monumental de Prentice repose sur l'étude de 182 caractères, presque tous tirés de la morphologie comparée des adultes, et quelques-uns de la morphologie larvaire. Il prend en compte toutes les tribus et soustribus des anciens Sphecidae, ainsi que le genre Heterogyna, plusieurs représentants des Apidae et quelques espèces appartenant à d'autres familles d'Aculéates. La plupart des analyses de parsimonie ont été conduites à partir d'une matrice de caractères incluant 42 taxa et un ancêtre hypothétique dont presque tous les caractères sont codés 0 (état plésiomorphe supposé). La fig. 1 représente, sous une forme simplifiée, l'un des cladogrammes obtenus par Prentice, correspondant à l'une des hypothèses phylogénétiques préférées. L'examen du cladogramme permet de dégager les résultats essentiels que voici.

1. Les Apidae et tous les taxa qui composaient les Sphecidae (au sens de BOHART \& MENKE, 1976) forment un ensemble monophylétique auquel on attribue maintenant le nom d'Apoidea. L'ancien taxon des Sphecidae, qui apparaît comme un groupement paraphylétique, ne peut être retenu comme une famille distincte. Toutefois, pour des raisons de commodité et pour se conformer à l'usage qui distingue les guêpes (anciens Sphecidae) et les abeilles 
(anciens Apidae), l'habitude a été prise par les auteurs de langue anglaise de désigner les anciens Sphecidae sous le nom de "apoid wasps", expression que nous traduisons en français par "Apoïdes sphéciformes" ou simplement "Sphéciformes", étant entendu qu'il s'agit seulement d'une expression vernaculaire.

2. Les Ampulicidae (Ampulicinae sensu BOHART \& MENKE, 1976) apparaissent comme un groupe monophylétique occupant une position basale au sein des Apoidea.

3. La position des Heterogynaidae (famille incluant l'unique genre Heterogyna) au sein des Apoidea reste controversée. Dans le cladogramme figuré ici, les Heterogynaini forment le groupe frère des Ampulicidae (Dolichurini + Ampulicini), mais d'autres analyses les montrent soit comme un groupe basal parmi les Apoidea, soit comme groupe-frère de l'ensemble des Crabronidae (pris dans un sens élargi) + Apidae, ou encore comme groupe-frère de l'ensemble des Sphecidae (s. str.) + Crabronidae + Apidae. Une discussion sur la place des Heterogynaidae a été présentée par OHL \& BLEIDORN (2006) (voir ci-dessous).

4. L'ancien groupe des Sphecinae, élevé au rang de famille, forme un clade comprenant notamment les Sceliphrini, Sphecini et Ammophilini.

5. Les Apidae s'intercalent entre les groupes précédents et un ensemble de lignées qui incluent toutes les autres tribus des anciens Sphecidae. Ce vaste ensemble monophylétique est désigné sous le nom de Crabronidae, taxon pris dans un sens très élargi par rapport à l'ancienne sousfamille des Crabroninae.

6. Les Astatini apparaissent comme une lignée isolée (élevée au rang de sous-famille des Astatinae) placée à la base des Crabronidae (sensu novo). Le rapprochement supposé des Dinetini et des Astatini, tel qu'il avait été admis par BoHART \& MENKE (1976) et par d'autres auteurs, est rejeté par les analyses cladistiques qui, au contraire, présentent les Dinetini comme le groupe frère d'un ensemble constitué par les anciens Larrinae et Crabroninae.

7. Les Alyssonini, Nyssonini et Bembicini (incluant les Gorytina, Handlirschina, Stizina, Bembecinina et Bembicina) forment ensemble un clade qui correspond à l'ancienne sousfamille des Nyssoninae, moins les Mellini. Ces derniers sont à rapprocher des Dinetini, à la base de l'ensemble des anciens Larrinae et Crabroninae.

8. Les Psenini et Pemphredonini restent groupés dans la sous-famille des Pemphredoninae, mais s'y ajoutent les Entomosericini, dont la position phylogénétique a été souvent discutée.

9. Les Philanthinae (Philanthini + Cercerini + Pseudoscoliini) apparaissent comme le groupe frère des Pemphredoninae.

10. Une dernière lignée comprend, outre les Mellini et Dinetini, les tribus précédemment groupées en deux sous-familles, les Larrinae et les Crabroninae. Les analyses de parcimonie indiquent que l'ancienne sous-famille des Larrinae est en réalité un groupe paraphylétique 
rapproché des Oxybelini et Crabronini. Le nom de Crabroninae, pris dans un sens élargi, est proposé pour grouper les anciens Larrinae et les anciens Crabroninae.

Les analyses cladistiques conduites par PRENTICE (1998) l'ont conduit à proposer une classification des Apoidea qui est reproduite ici, sous une forme simplifiée ( $c f$. annexe).

La classification des Apoidea retenue par PULAWSKI (Catalogue de 2007, accessible sur Internet) ne diffère de celle de Prentice que par quelques changements dans l'ordre de présentation des diverses lignées. Ainsi, au sein des Crabronidae (s. l.), les sous-familles sont appelées dans l'ordre suivant : Astatinae, Bembicinae, Crabroninae, Dinetinae, Mellinae, Pemphredoninae et Philanthinae.

L'étude de OHL \& BLEIDORN (2006) porte principalement sur la position phylogénétique des Heterogynaidae. Les analyses cladistiques présentées par ces auteurs utilisent des données moléculaires, les séquences d'un gène nucléaire, l'opsine à grande longueur d'onde. Ces analyses aboutissent à des résultats différents des précédents, en plaçant les Heterogynaidae au sein des Crabonidae (sensu Prentice), et non dans une position basale proche des Ampulicidae et des Sphecidae (s. str.). Dans un autre article, LOHRMANN et al. (2006) indiquent brièvement les résultats d'une étude préliminaire basée sur les séquences de deux gènes, la cytochrome-C-oxydase sous-unité 1 et la rhodopsine à grande longueur d'onde. Selon cette étude, les Ampulicidae et les Sphecidae s. str. (Sphecinae sensu Bohart \& Menke) forment un monophylum. Par contre la monophylie des Bembicinae et celle des Crabronidae (sensu Prentice) ne sont pas soutenues. Les Heterogynaidae semblent former un groupe dérivé parmi les Crabronidae. Enfin certains résultats placent les Philanthinae comme groupe-frère des Apidae et pourraient justifier leur désignation comme famille (Philanthidae); mais dans ce cas, ils formeraient avec les Apidae la superfamille des Apoidea sensu novo. Toutefois ces résultats provisoires devront être confirmés par une étude plus approfondie prenant en compte un échantillonnage plus élevé de taxa et un plus grand nombre de gènes.

Parmi les publications récentes sur les Apoïdes sphéciformes, on peut citer l'article de OHL (2007) qui fournit une mise au point sur les différentes stratégies de comportement des guêpes fouisseuses, en particulier sur les divers types de nidification et de parasitisme. Un autre article de OHL \& ENGEL (2007) concerne l'histoire des Aculéates fossiles, qui admet une origine commune aux Sphéciformes et aux Abeilles, la divergence entre les deux lignées remontant au début du Crétacé, il y a environ 125 millions d'années.

\section{p. 38 à 41 . Publications nouvelles sur la faune de France et des autres pays d'Europe.}

Dans la liste ci-dessous, les publications sont citées par pays et par ordre alphabétique des noms d'auteurs. Le détail des références figure dans la bibliographie donnée en fin d'article.

Faune de France et régions limitrophes. BASSET (2001) ; CARTIER (2000) ; CRETIN (1999); LeClerCQ \& BARBIER (1993); MAgdalou (2006a, b) ; SCHMID-EgGer (2001); VAGO (2005a, b).

Faune de divers pays d'Europe. DOLLFUSS (2006) ; DREWES (2003) ; LECLERCQ (1993).

- Allemagne. Pour l'ensemble de la faune des Sphecidae (s. l.) d'Allemagne, on dispose maintenant de deux ouvrages parus dans la collection "Die Tierwelt Deutschlands" : BLÖSCH (2000) qui traite des Sphecidae s.str. et des Crabronidae ; et JACOBS (2007) qui donne une clé d'identification des espèces. Autres références : ARENS \& HARTMANN (2004); BLÖSCH (1996, 1998, 2003) ; BURGER et al. (1998) ; CÖLlN \& JAKUBZIK (1999, 2000, 2008) ; CÖLlN et al. (2003) ; DATHE \& BlANK (2004); DrewES (1998); DunK V. D. \& AMON (2002); ERHARDT (1999) ; ESSER \& CÖLLN (2002); ESSER \& JAKUBZIK (2003); ESSER et al. (2004a, b) ; FlÜGEL (2003) ; FreUnDT \& ILlmer (2003) ; HAESELER (1997, 2001, 2003, 2005); HAESELER \& RITZAU (1998); HEIDE V. D. \& METSCHER (2003); HERRMANN (1999); JACOBS (1999, 2000, 2005a) ; JAKUBZIK (1996) ; JAKUBZIK \& CÖLLN (1993, 1996, 2005) ; JAKUBZIK et al. (1998) ; KRAATZ (2005) ; KRAUS \& FLOREN (2002) ; KUHLMANN $(1993,2000)$; KuHL- 
MANN \& QUEST (2003) ; KUHLMANN et al. (1999); KULA \& TYRNER (2003); MANDERY (2005) ; MAUSS \& SCHINDLER (1998); MAUSS et al. (2000); OHL (2001, 2003); QUEST \& KUHLMANN (2005) ; REDER $(2004,2005)$; RIEMANN $(1995,1997,1999)$; SAURE \& DÜRRENFELD (1995) ; SCHLÜTER (2002); SCHMID-EGGER (1994, 1996, 2000, 2001); SCHMID-EGGER \& NiEHUIS (1997); SCHMID-EGGER, RISCH \& NIEHUIS (1995); SCHMIDT \& SCHMID-EGGER (1997) ; SMISSEN V. D. (1993, 1998) ; SÖRENSEN et al. (1999) ; SORG \& WOLF (1993) ; STANDFUSS \& STANDFUSS (2006a); STOLLE et al. (2004); THEUNERT (1994 à 2008) ; TISCHENDORF (2001) ; TISCHENDORF \& FROMER (2004); TISCHENDORF \& TREIBER (2003); WEBER (1998) ; WICKL (1999); WICKL \& WiCKL (1994); WITT (1996) ; WOLF (1993, 1995, 2001, 2003) ; WOYDAK (1996) ; ZISKA (2002).

- Autriche. Aistleitner (2004) ; Dollfuss $(1994,2004)$; Dollfuss et al. (1998) ; GUSENLEITNER (1995 à 2005); SCHWARZ (1999); ZETTEL (2000, 2004); ZETTEL et al. (2001, 2008).

- Belgique. LitT (2002) ; PAUly (1999); PeTIT (1997, 1998).

- Bulgarie. Georgiev \& Ljubomirov (2000), Georgiev et al. (2004); IVANOV \& LJUBOMIROV (2001); JACOBS (2005b) ; LJUBOMIROV (1999, 2000, 2001, 2006) ; STOYANOV \& LJUBOMIROV (1997).

- Estonie. LUIG \& VoOlma (2000).

- Finlande. KOPONEN (1994); VIKBERG (1999).

- Grande-Bretagne. ARCHER (1993 à 2008); BEAVIS (2000, 2001, 2005) ; DENTON (1998) ; HARVEY (1996); HARVEY \& Plant (1996); PRICE (2001).

- Grèce. GiACHINO et al. (2000) ; LJUBOMIROV (2006) ; LECLERCQ (2007) ; STANDFUSS $\&$ STANDFUSS (2006b).

- Hongrie. JÓZAN (1993, 1995) ; PAPP \& JÓZAN (1995).

- Italie. GAYUBO \& BORSATO (1994) ; GENERANI et al. (1998, 2003) ; LUCHETTI (1993); Mochi \& LuCHETTI (1993) ; NEgRISOlO (1995a, b) ; NEGRISOlO \& PAGLIANO (1993) ; PAGLIano (2003) ; Pagliano \& Negrisolo (2005) ; PAgliano \& Pesarini (1995); Pagliano \& SCARAMOZZINO (1995); SCHMID-EGGER (2003); TOMARCHIO \& TURRISI (2006).

- Lituanie. BUDRYS (2001).

- Luxembourg. CUNGS \& JAKUBZIK (2001) ; FEITZ et al. (2001, 2003, 2006) ; FELTON \& SCHNEIDER (1994); JUNK et al. (1994) ; KLEIN (1996); SCHNEIDER \& CARRIÈRES (2004).

- Pays-Bas. LefeBer (1998); PEeTERs et al. (2004); VAN DER MEer (2002).

- Péninsule Ibérique. BAÑOS-PICON et al. (2007) ; CRUZ-SÁNCHEZ et al. (2005) ; GAYUBO et al. (1993, 1999, 2000, 2002, 2004a, b, 2006, 2008) ; GONZÁLEZ et al. (1998, 1999, 2000, 2003) ; NiEVES-AldREY et al. (2003); SMIT (2000); TORMOS et al. (1994); TORREGROSA et al. (1993).

- Pologne. Celary (1998) ; KowalczyK (1994); KowalcZyK \& KrZePtowski (1995) ; KOWALCZYK et al. (2002) ; PAWLIKOWSKI \& KRUSZYNSKI (1996); SKIBINSKA (1995, 1999, 2001) ; SZCZEPKO \& KOWALCZYK (2001, 2004); WiSNIOWSKI (1993, 2000, 2002, 2004, 2005a, b) ; WISNIOWSKI \& KOWALCZYK (1998); WISNIOWSKI \& WERSTAK (2003).

- République Tchèque et Slovaquie. DOLlfUSS (2004); KULA \& TYRNER (2003); LUKAS \& LISKA (2005); PADR \& LUKAS (1994); SRBA (1999); VEPREK \& STRAKA (2007).

- Suède. ABENIUS \& LARSSON (2006); NILSSON (1992).

- Suisse . AMiET \& MoretTi (2002) ; LitT (2004) ; SALZMANN-WANDELER (1998a, b) ; SALZMANN-WANDELER \& REZBANYAI-RESER (1999, 2001, 2004); VERNIER (1994) ; ZEHNDER \& ZETTEL (1999).

- Ukraine. ElLIGSEN (1998) ; GOROBCHISHIN (1993, 1995, 1996) ; VOBLENKO et al. (1996).

p. 41. Rectifications aux données chorologiques pour les autres pays d'Europe. Pour la péninsule Ibérique, lire : AsIS \& JIMENEZ $(1987,1988)$ [au lieu de : 1986, 1988] ; pour la Scandinavie, lire : NILSSON (1989) [au lieu de NILSON (1989)]. 
p. 45. La clé des genres d'Europe doit être complétée par l'adjonction du genre Pseudomicroides, distinct de Belomicroides. Voir plus loin le complément de la page 233 (Tribu des Oxybelini).

p. 50. Plusieurs erreurs se sont glissées dans la légende de la fig. 21. Pour la fig. 21b, lire : Prionyx [au lieu de Isodontia mexicana]. Pour les dessins 21d, $21 \mathrm{f}$ et $21 \mathrm{~h}:$ lire Palmodes occitanicus [au lieu de occitanus].

p. 51. A la fin des $\S 34$ et 34bis, mettre en gras les chiffres de renvoi 35 et 36 .

p. 59. A la fin de ligne 2 : supprimer un tiret après [Genre Prosopigastra]. A la fin du premier couplet 73, mettre en gras le chiffre de renvoi 74.

p. 61. La sous-famille des Crabroninae, telle qu'elle est traitée dans la présente faune, inclut seulement les tribus des Crabronini et Oxybelini alors que dans la classification proposée par PRENTICE (1998), le terme de Crabroninae Latreille est pris dans un sens plus large (voir plus haut). Une revue des données publiées sur la biologie de différentes espèces a été fournie par KAZENAS (2001).

p. 71. Crabro lapponicus Zetterstedt. Une femelle capturée le 19.07.2002 en Autriche : Kärnten (GUSENLEITNER, 2002) ; espèce trouvée aussi en République Tchèque et en Slovaquie (DOLLFUSS, 2004).

p. 72. Crabro alpinus Imhoff. Signalé pour la première fois de Bulgarie (LJUBOMIROV, 1999) et du nord de l'Espagne: val d'Aran, proche de la frontière française (GAYUBO, GONZÁLEZ et al., 2002). Observé dans diverses provinces d'Italie du nord et dans les Abruzzes (PAGLIANO \& Negrisolo, 2005). Dans la vallée des Merveilles (Alpes-Maritimes), 2300 m, 23.07.1998, 1 femelle, et 1500 m, 9.08.2004, 1 femelle (J. Smit leg.).

p. 72. Crabro ingricus (F. Morawitz). Signalé pour la première fois de Pologne (SKIBINSKA, 2001). Jamais retrouvé en Suisse depuis 1933. En France à notre connaissance pas de capture depuis celle de 1966 à Annot (Alpes-de-Haute-Provence).

p. 73. Crabro cribrarius (Linné). Nouveau département français : Loire, Veauche.

p. 76. Crabro peltarius (Schreber). Nouveaux départements français pour C. peltarius peltarius : Ain, Sermoyer, bac jaune, 31.07.1995 (J. Hamon leg.). - Aisne, Rocourt-SaintMartin, 21.06.2007, 1 mâle (E. Gros leg.). - Eure-et-Loir, Thiron, 8.08.1985, 1 mâle ; Pontgouin 20.07.1986, 1 femelle (A. Adamski leg.). - Gironde, marais du Cla (Hostens) (BASSET, 2001). - Sarthe, Savigné-sur-le-Lude, mai et juin 1992, 2 femelles, 1 mâle (L. Faillie leg.). Sousespèce bilboensis : signalée pour la première fois de Sicile (SCHMID-EGGER, 2003). Nouveau département français pour C. p. bilbaoensis : Lot-et-Garonne, Mezin, 11.05.1992, 2 mâles (J.-Cl. Teulou leg.).

p. 78. Crabro peltatus Fabricius. Observé au mont Pirin, en Bulgarie, ce qui semble marquer le point le plus méridional de sa répartition dans la péninsule Balkanique (DoLLFUSS, 2004 ; JACOBS, 2005b).

p. 78. Crabro pugillator A. Costa. Trouvé dans le sud de la Bulgarie, monts Rhodope (LJUBOMIROV, 2006).

p. 79. Crabro scutellatus (Scheven). Nouveaux départements français : Lot-et-Garonne, Andiran, sablière, juin 1996 (J.-Cl. Teulou). - Moselle, Folschviller (G. Jacquemin leg.). Pas-de-Calais, Hardelot-plage (Y. Barbier leg.).

p. 81. Crabro loewi Dahlbom. L'espèce a été retrouvée plus récemment dans le site découvert par Chevin (Y. Barbier, com. pers.). 


\section{p. 82. GENRE CROSSOCERUS Lepeletier \& Brullé}

Dans sa clé des espèces américaines, LECLERCQ (2000) appelle plusieurs espèces qui se trouvent aussi en Europe : C. acanthophorus (Kohl), elongatulus (Vander Linden), leucostoma (Linné), nigritus Lepeletier \& Brullé, wesmaeli (Vander Linden); en outre, avec des données éthologiques inédites : C. annulipes (Lepeletier \& Brullé), barbipes (Dahlbom) et tarsatus (Shuckard). Dans une révision des espèces paléarctiques décrites par Tsuneki, NEMKOV (2004) fournit des informations (synonymies, distribution) sur plusieurs espèces qui se trouvent en Europe : C. capitosus (Shuckard), cetratus (Shuckard), cinxius (Dahlbom), heydeni Kohl, nigritus (Lepeletier \& Brullé) et styrius (Kohl). LECLERCQ (2007) ajoute à l'inventaire des Crossocerus de Grèce, qui comporte actuellement 16 espèces et sous-espèces, une espèce nouvelle, Crossocerus (s. str.) epiri (voir plus loin). Enfin LECLERCQ (2009) présente une nouvelle clé des sous-genres et un catalogue à jour des espèces d'Asie.

p. 85 à 91. Clé des espèces du genre Crossocerus, femelles.

Modification proposée de plusieurs couplets :

26. Grand éperon du tibia 1 brun-noir... Sillon médian de l'enclos propodéal plus large en avant, plus ou moins ovale (fig. 34b). Tibia 2 souvent avec seulement un anneau basal jaune

tarsatus (Shuckard)

- $\quad$ Grand éperon du tibia 1 jaune pâle... Sillon médian de l'enclos propodéal moins large, en triangle (fig. 34c). Tibia 2 souvent avec une coloration jaune plus étendue varus Lepeletier \& Brullé

27. Bord postérieur du scutum avec de courtes stries longitudinales très distinctes (moins distinctes chez denticoxa)...

30. Fossettes orbitales indistinctes. Clypéus largement jaune sur sa moitié antérieure. Collare et scutellum non tachés de jaune. Aire pygidiale plus sombre, au plus son tiers apical ferrugineux

16. denticoxa (Bischoff)

- Fossettes orbitales distinctes. Clypéus avec deux petites taches jaunes. Collare et scutellum tachés de jaune. Aire pygidiale bicolore : sa partie distalement largement ferrugineux clair

25. wesmaeli (Vander Linden) var.

Ressemble à

toledensis Leclercq

35. Largement taché de jaune

[elongatulus trinacrius (Beaumont)]

Mandibule entièrement ferrugineux sombre. Pas de jaune aux pattes, sauf à la face inférieure du tibia 1 et un petit point basal aux tibias 2 et 3

[italicus Beaumont]

36a. Fossettes orbitales absentes ou indistinctes. Clypéus jaune dans toute ou presque toute sa moitié antérieure. Lobe pronotal jaune, mais collare et scutellum non tachés .... denticoxa (Bischoff)

- $\quad$ Fossettes orbitales distinctes

p. 93 et 95. Dans la clé des mâles de Crossocerus, modifier deux strophes comme suit :

7. Basitarse 1 élargi et aplati (non subrectangulaire), nettement plus large que le basitarse 2 .......... 8

- Basitarse 1 beaucoup moins large, non ou à peine plus large que le basitarse 2, ses bords toujours parallèles

11. Basitarse 1 fortement dilaté... Tibia 1 plus élargi vers l'extrémité, avec des soies assez longues dans son quart distal, son éperon jaune

5. annulipes (Lepeletier \& Brullé) Basitarse 1 moins largement dilaté... Tibia 1 moins élargi, avec des soies beaucoup plus courtes dans son quart distal, son éperon sombre 23. tarsatus (Shuckard)

p. 96. Pour introduire l'espèce italicus, dont le mâle est maintenant connu, modifier comme suit le $\S 23$ :

23. Tergite VII large, transversal, presque tronqué en arrière (fig. 35 h). Collare épais, ses angles marqués, parfois fortement

- Tergite VII en demi-cercle. Collare en arc surbaissé. Fémur 2 non élargi à la base, son épaisseur maximum vers le milieu

23a. Fémur 2 brusquement élargi dès la base (fig. 37 b). Métapleure lisse, en tout cas sans strioles distinctes. Mésopleure à ponctuation régulière, sans rugosités. Plusieurs sous-espèces 
Fémur 2 non élargi à la base, son épaisseur maximum vers le milieu. Métapleure striolée.

Mésopleure à ponctuation plus forte, compliquée de strioles avant et après le sillon épisternal

[italicus Beaumont]

p. 98. Crossocerus assimilis (F. Smith). Signalé de Finlande (VIKBERG, 1999) et d'Estonie (Luig \& VoOlma, 2000). Quelques captures en Roumanie, Slovénie, Slovaquie, République Tchèque et Bulgarie (LECLERCQ, 1993 ; PADR \& LuKAS, 1994 ; LJUBOMIROV, 1999 ; JACOBS, 2005b ; DOLLFUSS, 2006). Nouveaux départements français : Ain, Tenay, les Hôpitaux, $800 \mathrm{~m}$, 11.07.2002, 1 mâle (J. Smit leg.). - Isère, Saint-Laurent-en-Beaumont, 21.07.1991, 1 femelle (A. Moussa leg.). - Nord, Wasnes-au-Bac, 15.06.1999, 2 femelles (VAGO, 2005a). - Puyde-Dôme, col de Guéry (Orcival) et Puy de Cliergue (Mont-Dore), 31.07 .1992 (Y. Barbier leg.). - Haut-Rhin, Munster, 900m, 28.07.1999, 1 femelle, et 1000 m, 9.07.2002, 1 mâle (J. Smit leg.).

p. 99. Crossocerus congener (Dahlbom). JACOBS (1999) précise les différences morphologiques qui doivent permettre de distinguer les petites femelles de $C$. podagricus de celles de $C$. congener. Chez $C$. podagricus, aire dorsale du propodeum le plus souvent limitée vers l'arrière par un sillon distinctement crénelé; aire pygidiale nettement trilobée, fortement élargie en avant, ses côtés concaves avec une étroite bordure éclaircie. Chez C. congener, aire dorsale du propodeum jamais limitée en arrière par un sillon crénelé ; aire pygidiale seulement un peu élargie en avant, ses côtés presque droits, assez largement brun clair. THEUnERT (2007a) relate des observations faites en Allemagne (Basse-Saxe); les nids se trouvent, parfois en pleine ville, dans du bois mort, dur ou pourri, provenant de divers arbres feuillus et d'arbustes. C. congener a été récemment signalé de Bulgarie, de la République Tchèque et de Roumanie (LJUBOMIROV, 1999 ; DOLLFuSS, 2006), d'Estonie (LUIG \& VoOLMA, 2000), ainsi que du Luxembourg (FEITZ et al., 2003, 2006 ; SCHNEIDER \& CARRIÈRES, 2004). En France, nouveau pour les départements du Loiret (A. Larivière leg.), de l'Isère, Grenoble, mai 1976, 1 mâle (A. Moussa leg.) et du Pas-de-Calais (VAGO, 2005a).

p. 100. Crossocerus podagricus (Vander Linden). Voir le paragraphe ci-dessus, référence à JACOBS (1999). Signalé pour la première fois d'Estonie (LuIG \& VoOLMA, 2000) et de Sicile (SCHMID-EGGER, 2003). Cité de Bulgarie, Croatie, Roumanie, République Tchèque, Slovaquie et Ukraine, ainsi que du Maroc et de la Turquie (Dollfuss, 2006). Capturé aussi en Grèce continentale (GIACHINO et al., 2000; STANDFUSS \& STANDFUSS, 2006b). Nouveaux départements français : Aisne (VAGO, 2005a). - Cantal, lac de Cregut (F. Durand leg.). - Sarthe, Saint-Cosme-en-Vairais, jardin, piège Malaise, 21.08.1993, 1 femelle, 1 mâle (C. Dussaix leg.).

p. 102. Crossocerus vagabundus (Panzer). Cité de Grèce et d'Estonie (LECLERCQ, 1993 ; LuIG \& VoOlMA, 2000), de Bulgarie (LJUBOMIROV, 2000, 2001) et d'Espagne, Sierra de Guadarrama (GAYUBO, GONZALEZ et al., 2002 ; NIEVES-ALDREY et al., 2003; GAYUBO, NIEVES-ALDREY et al., 2004a). Connu de plusieurs provinces du nord de l'Italie, ainsi que des Abruzzes, du Latium et de Calabre (PAgliano \& Negrisolo, 2005). Aussi en Algérie et Tunisie (LeClerCQ, 1993). Quelques mentions récentes en France, dans le département du Nord, forêt de SaintAmand, 19.06.1999, 1 mâle (VAGO, 2005a) (l'espèce avait déjà été signalée du département du Pas-de-Calais par CAVRO en 1950), et dans les Pyrénées-Orientales : Réserve Naturelle de la Massane (MAgdalou, 2006a). Autres départements français : Ardèche, Les Vans ( $L$. Leseigneur leg.). - Bouches-du-Rhône, Eygalières (R. Fonfria leg.).

p. 103. Crossocerus annulipes (Lepeletier \& Brullé). Cité d'Estonie (LuIG \& Voolma, 2000). Nouveau département français : Sarthe, Saint-Cosme-en-Vairais, jardin, piège jaune 21.08.1993, 1 femelle (C. Dussaix leg.).

p. 105. Crossocerus barbipes (Dahlbom). Cité de Bulgarie (LuUbomirov, 2001). Nouveau département français : Ariège, Goulier, entre 1000 et 2400 m., juillet 1984, 2 mâles (B. Chaubet leg.) 
p. 106. Crossocerus capitosus (Shuckard). Trouvé en Estonie (Luig \& VoOLma, 2000). En Italie du nord (Trentin-Haut-Adige, Piémont, Emilie-Romagne), aussi en Toscane, Ombrie, Marches et Abruzzes (PAGLIANO \& NEGRISOLO, 2005). En Espagne du Nord, signalé récemment de la province des Asturies (GAYUBO, GONZÁLEZ et al., 2006). Nouveau département français : Var, Montauroux, 10.05.1992, 1 mâle (C. Cocquempot leg.). Autrefois cité de deux localités, l'une dans le département du Nord, l'autre dans le Pas-de-Calais (CAVRO, 1950 ; VAGO, 2005a).

p. 107. Crossocerus cetratus (Shuckard). Cité d'Estonie (LUIG \& Voolma, 2000). Nouvelle capture en Grèce (GIACHINO et al., 2000) et en Sicile, mont Etna, 1600 m (TOMARCHIO $\&$ TURRISI, 2006). Nouveaux départements français : Alpes-Maritimes, Castérine, $1500 \mathrm{~m}$, 24.07.2001, 1 femelle (J. Smit leg.). - Nord, Wasnes-au-Bac, 12.06.1999, 1 femelle (VAGO, 2005a). - Sarthe, Saint-Cosme-en-Vairais, jardin piège jaune, 21.08.1993, 1 femelle $(C$. Dussaix leg.).

p. 108. Crossocerus cinxius (Dahlbom). Cité pour la première fois d'Estonie (LUIG \& VoOlma, 2000) et de Bulgarie (JACOBS, 2005b). En France, le département des Hautes-Alpes est à supprimer, car il résulte d'une erreur d'identification. Nouveau département : Haut-Rhin, Munster, 900 m, 3.07.2000, 1 femelle (J. Smit leg.).

p. 109. Crossocerus heydeni Kohl. Cité pour la première fois de Pologne (WISNIOWSKI \& KowALCZYK, 1998), du Vorarlberg en Autriche (GuSENLEITNER, 2000), d'Estonie (LUIG $\&$ VoOlma, 2000) et du Luxembourg (FeITZ et al., 2003), mais semblait inconnu en Belgique et aux Pays-Bas (SCHNEIDER \& CARRIERES, 2004) jusqu'à la capture de 2 mâles sur tronc mort en Belgique à Doische le 13.09.2007 (Y. Barbier leg.). Le catalogue d'Allemagne (OHL, 2003) signale cette espèce dans 6 Länder : Brandenburg, Nordrhein-Westfalen, Thüringen, RheinlandPfalz, Baden-Württemberg et Bayern. A notre conniassance, aucune capture récente en France.

p. 111. Crossocerus leucostoma (Linné). Capture d'un mâle en Autriche, dans le Burgenland, 1.05.1997 (Dollfuss et al., 1998 ; carte 115). OHL (2003) cite sa présence dans toutes les provinces d'Allemagne. En Italie, l'espèce est citée de cinq provinces du nord, ainsi que Toscane, Campanie et Calabre (PAgliano \& Negrisolo, 2005). Aucune citation récente d'Espagne. Captures récentes en France : Haute-Loire, Les Estables, 1580 m, 23.07.1994, 1 femelle (J. Hamon leg.). - Haut-Rhin, Munster, 1100 m, 9.07.2002, 1 femelle, et $2980 \mathrm{~m}$, 1.07.2004, 1 femelle, 1 mâle (J. Smit leg.). - Savoie, Bonnevaux, 1350 m, 16.06.1996, 1 femelle (J. Hamon leg.).

p. 112. Crossocerus megacephalus (Rossi). Nidification et stockage des proies : PIPER (2007). Signalé de Hongrie (JÓZAN, 1993), de Bulgarie (LJUBOMIROV, 1999 ; JACOBS, 2005b), d'Estonie (Luig \& VoOlma, 2000), de République Tchèque (Kula \& TYRnER, 2003 ; DollFUSS, 2006), de Suisse (SALZMANN-WANDELER, 1998a ; SALZMANN-WANDELER \& REZBANYAIRESER, 1999) et de Grèce (GIACHINO et al., 2000). Nouveaux départements français : Aude, Antugnac, 14.05.1993, 1 femelle ; Salvezines (T. Noblecourt leg.). - Puy-de-Dôme, La RocheNoire (F. Durand leg.).

p. 113. Crossocerus nigritus (Lepeletier \& Brullé). Cité d'Autriche (BURGER et al., 1998 ; Dollfuss et al., 1998 ; SCHWARZ, 1999), de Bulgarie (GEORGIEV \& LJubOMIROV, 2000 ; GeORGIEV et al., 2004), des Pays-Bas et du Luxembourg (LEFEBER 1998 ; PETIT 1998 ; SCHNEIDER \& WAHIS, 1998 ; CUNGS \& JAKUBZIK, 2001 ; LITT, 2002 ; SCHNEIDER \& CARRIÈRES, 2004) et de Suisse (SAlZMANn-W ANDEler \& ReZBANYAi-ReSER, 1999, 2001, 2004 ; AMIET \& MoretTI, 2002). Cité d'Italie du nord et des Abruzzes (PAgliano \& Negrisolo, 2005). A notre connaissance, aucune citation récente d'Espagne. Nouveau département français : HautesAlpes, Haute vallée du Guil (F. Fonfria leg.).

p. 115. Crossocerus styrius (Kohl). Nouveau pour la Bulgarie (LJUBOMIROV, 1999), pour l'Estonie (LUIG \& VoOLMA, 2000) et pour la province de Navarra en Espagne (GAYUBO, 
GonZÁlez et al., 2002). Cité de la République Tchèque (KulA \& TYRnER, 2003) et de Suisse (SALZMANN-WANDELER \& REZBANYAI-RESER, 2004). En France, nous n'avons connaissance d'aucune capture postérieure à celle faite en Haute-Savoie, Bossy/Frangy, 1 mâle éclos en mai 1990 (J. Steffan leg.).

p. 116. Crossocerus walkeri (Shuckard). Signalé pour la première fois en Estonie (LuIG \& Voolma, 2000). Nouveaux départements français : Alpes-de-Haute-Provence, Montagne de Lure, Cruis, 16.06.1981, 1 mâle (Dollfuss, 2006). - Puy-de-Dôme, Mirefleurs, 5.06.1995, 1 femelle (F. Durand leg.).

p. 117. Sous-genre CROSSOCERUS s. str. A la fin du premier paragraphe, ajouter : LECLERCQ (2007) a décrit une nouvelle espce de Grèce, Crossocerus (s. str.) epiri, proche de $C$. ovalis, $C$. varus et $C$. tarsatus. Chez toutes ces espèces, les mâles possédent une aire pygidiale rebordée et ponctuée, caractéristique de l'ancien genre Stenocrabro, abandonné à la suite de PATE (1944) pour qui le caractère considéré justifierait tout au plus une "section". PRENTICE (1998) admet qu'une plaque pygidiale chez le mâle des Sphéciformes a dû apparaître de nombreuses fois au cours de l'évolution des "apoid wasps" et qu'elle ne peut ainsi constituer un caractère d'intérêt phylogénétique. Pour Leclercq, au contraire, la présence d'une aire pygidiale rebordée et ponctuée chez le mâle pourrait être considérée comme un caractère plésiomorphe, "qui certes a été l'objet de maintes réversions dans les lignées qui sont passées de la nidification dans le sol (primitive) à la nidification dans le bois".

p. 117. Crossocerus denticoxa (Bischoff). Deux nouvelles captures en Allemagne, dans le Baden-Württemberg, l'une en 1981, l'autre en 1992 (SCHMIDT \& SCHMID-EGGER, 1997). En France, l'espèce n'était connue que d'après une femelle prise dans le Bas-Rhin en 1959 (LECLERCQ, 1991d), déjà signalée dans le vol. 1 de la présente Faune. Une nouvelle femelle a été prise dans un département éloigné, le Tarn-et-Garonne, dans une sablière à Andiran, le 17.06.1996 (J.-Cl. Teulou leg. ; J. Leclercq det.).

p. 119. Crossocerus denticrus Herrich-Schaeffer. Non retrouvé en Allemagne depuis 1946 (SCHMIDT \& SCHMID-EGGER, 1997). Espèce nouvelle pour l'Autriche, d'après la capture d'une femelle à Oberau, Steiermark, le 6.06.1994 (GuSENLEITNER, 2003). Nouveau département français : Landes, Saint-Julien-en-Born, 1996 (Y. Barbier leg.).

p. 120. Crossocerus distinguendus (A. Morawitz). Nouveau pour le Vorarlberg en Autriche (GuSENLEITNER, 1999), signalé aussi de Bulgarie (STOYANOV \& LJubOMIROV, 1997 ; LJuboMIROV, 2001). Atteint $2300 \mathrm{~m}$ dans les Alpes-Maritimes, Castérine, 23.07.1998, 1 femelle (J. Smit leg.).

p. 120. Crossocerus elongatulus (Vander Linden). La sous-espèce trinacrius Beaumont trouvée dans les îles italiennes de Lampedusa et Pantelleria (PAGLIANO, 2003). Nouveaux départements français : Sarthe, La Flèche, jardin, 23.06.1992, 1 mâle (L. Faillie leg.). - Loiret, Nogent-sur-Vernisson, 2.06.1990, 1 mâle (A. Larivière leg.).

p. 125. Ajout : Crossocerus epiri Leclercq, 2007. Espèce nouvellement décrite de Grèce : Ionninon, E. Metsovon, col de Katara, 1700m, 31.07.1983, 1 femelle et 2 mâles (voir ci-dessus le paragraphe consacré au sous-genre Crossocerus s. str.).

p. 125. Crossocerus exiguus (Vander Linden). Cité pour la première fois de Suède, province de Halland (ABENIUS \& LARSSON, 2006). Nouveaux départements français : Isère, Grenoble 900 m, 30.07.2002, 1 femelle (J. Smit leg.). - Nord, Cambrai et forêt de Saint-Amand, plusieurs captures notamment en juin 1999 et 2000 (VAGO, 2005a).

p. 125. Crossocerus italicus Beaumont. Référence à ajouter : LECLERCQ, 2007 (description du mâle jusqu'ici inconnu, de Grèce). Espèce jusqu'ici considérée comme endémique des Abbruzes (Italie) (PAGLiAno \& NEGRISOLO, 2005), mais dont les deux sexes ont été trouvés 
dans plusieurs localités de Grèce continentale, entre 1974 et 1983, à des altitudes comprises entre 1500 et $2500 \mathrm{~m}$ (LECERCQ, 2007).

p. 126. Crossocerus ovalis Lepeletier \& Brullé. Nouveau pour la Bulgarie (LJUBOMIROV, 1999). Cité d'Espagne, province de Soria, d'après la capture d'une femelle en juin, dans les années 1988 et 1990 (GAYUBO, GARCIA et al., 1999) ; l'espèce n'vait été précédemment signalée d'Espagne que par un mâle pris en juin 1906 à Madrid, et une femelle provenant également de la province de Madrid (LECLERCQ, 1971). Autrefois signalé du département du Var, environs de Fréjus, 1 femelle sur un chêne en octobre (BERNARD, 1935). Mais la détermination reste douteuse et l'espèce est considérée comme absente de Provence.

p. 127. Crossocerus palmipes (Linné). En Italie, trouvé en Vénétie, Piémont et Toscane (PAgliano \& Negrisolo, 2005).

p. 128. Crossocerus pullulus (A. Morawitz). Nouveau pour l'Autriche, Basse-Autriche, 26.08.1995, 1 mâle (GuSENLEITNER, 1996a). En Pologne, connu d'un petit nombre de localités de la côte baltique, mais trouvé aussi en 2003 dans les monts Beskides occidentaux, à la frontière de la Slovaquie (WISNIOWSKI, 2004).

p. 129. Crossocerus tarsatus (Shuckard). Trouvé dans un petit nombre de localités de Pologne depuis 1970 ; pris en 2003 dans le "Kraków-Wielun Upland" (WISNIOWSKI, 2004). En Italie, PAGLIANO \& Negrisolo (2005) citent $C$. tarsatus de 10 régions, dont la Sardaigne et la Sicile ; se référant à LECLERCQ (1993), ces auteurs admettent que c'est la sous-espèce richardsi qui est présente en Italie, ainsi qu'en Corse. Nouveau pour les Balkans, mais sans précision de sous-espèce : Bulgarie (LJUBOMIROV, 2001), Crête (Dollfuss, 2006) et Grèce : Thessalie (STANDFUSS \& STANDFUSS, 2006b). Trouvé pour la première fois en Roumanie, comté de Marmures, en juillet 2004 (LJUBOMIROV \& BAN-CALEFARIU, 2008). Nouveaux départements français : Alpes-de-Haute-Provence, montagne de Lure (DoLLFUSS, 2006). Lozère, Nasbinals (Godeau \& Patiny leg.). - Puy-de-Dôme, Orléat, octobre 1995, 4 femelles (F. Durand leg.). - Var, Gonfaron (P. Rasmont leg.).

p. 132. Crossocerus toledensis Leclercq. L'espèce, initialement décrite de Tolède, a été reprise dans les provinces de Valladolid et de Soria (GONZALEZ, GAYUBO \& TORRES, 1998, 1999 ; GAYUBO, GARCiA et al., 1999), ainsi qu'en Catalogne, à 40 km Tortosa, rivière Ebre, 1 mâle, 16.05.2003 (DOLlFuss, 2006).

p. 132. Crossocerus varus Lepeletier \& Brullé. Espèce observée ces dernières années dans plusieurs régions d'Espagne : Avila, Asturias, Madrid et Cantabria (GAYUBO, GonZÁLEZ et al., 2002, 2006 ; GAYUBO, NiEVES-ALDREY et al., 2004a) ; également en Andorre. Présent en Bulgarie et en République Tchèque (DOLlFuss, 2006 ; LJUBOMIROV, 2006).

p. 133. Crossocerus wesmaeli (Vander Linden). En France, retrouvé ces dernières années dans les départements du Nord et du Pas-de-Calais, et cité pour la première fois de la Somme, Le Crotoy, dunes, 4.08.1972 et 4.08.1975 (VAGO, 2005a).

p. 134. Crossocerus binotatus Lepeletier \& Brullé. Signalé pour la première fois en Estonie (Luig \& Voolma, 2000), en Bulgarie, captures de 1997 et 1998 (LJUBOMIROV, 1999), en Espagne, province de Navarra, non loin de la frontière française, captures en juillet et septembre 1995, et août 1996 ; aussi en Andorre en juillet 1993 (GAYUBO, GonZÁLEZ et al., 2002). Une femelle trouvée en août 2004 dans la province Cantabrique (GAYUBO, GONZÁLEZ et al., 2006). Nouveaux départements français : Haut-Rhin, Munster, 900 m, 3.07.2000, 1 femelle (J. Smit leg.). - Var, La-Garde-Freinet, 1993 (Y. Barbier leg.) et Montauroux, 10.05.1992, 1 mâle (C. Cocquempot leg.).

p. 135, ligne 9. Lire : tergites III-VI [au lieu de II-VI].

p. 136. Crossocerus dimidiatus (Fabricius). Deux données anciennes pour le nord de l'Espagne ont été retenues dans la carte 22 de LECLERCQ \& BARBIER (1993). DOLLFUSS (2006) 
signale la capture d'une femelle dans les Pyrénées de Catalogne à 1900 m, 23.08.1990, et de plusieurs exemplaires des deux sexes en République Tchèque. Nouveau pour la Bulgarie (LJUbOMIROV, 2001). Nouveaux départements français : Aisne, Soissons juin 1975 (VAGO, 2005a). - Alpes-Maritimes, Isola 2000, 24.07.1993, 1 mâle (C. Cocquempot leg.), Castérine 1550 m, 22.07.2001, 1 mâle (J. Smit leg.). - Ardèche, lac d'Issarles 1175 m, 24/25.07.1994, 1 femelle (J. Hamon leg.).

p. 137. Crossocerus subulatus (Dahlbom). Une nouvelle localité de Finlande, dans la région de Mikkeli, 1 femelle, 1.08.1978 (LECLERCQ, 1993). Signalé pour la première fois d'Estonie (Luig \& VOOLMA, 2000).

p. 137. Crossocerus quadrimaculatus (Fabricius). Observation éthologique à ajouter, faite en Italie centrale (L'Aquila) : celle d'un nid d'un Sphécide indéterminé, probablement C. quadrimaculatus, dont les larves sont parasitoïdes d'un Lépidoptère adulte du genre Zygena (ZILLI, 1991). Dans le nord-est de l'Angleterre, le Diptère Anisopodide Sylvicola genestralis a été signalé comme proie (BEAVIS, 2001). Nouveaux départements français : Alpes-Maritimes, pont du Loup, 23.08.1992, 1 femelle (C. Cocquempot leg.). - Cantal, à la limite du Puy-deDôme, lac de Crégut, 30.08.1992, 1 mâle (F. Durand leg.). - Haute-Savoie, embouchure de la Dranse (DrEwES, 2003). Nouvelles localités dans le Nord et le Pas-de-Calais (VAGO, 2005a).

p. 140. Crossocerus guichardi Leclercq. Espèce connue de France, retrouvée en Italie, Abruzzes (Pagliano \& NegRisolo, 2005) et en République Tchèque, 9.05.1974, 1 mâle (DOLLFUSS, 2006).

p. 141. Crossocerus acanthophorus (Kohl). Signalé de plusieurs provinces d'Espagne, en plus de celle de Gerona : Madrid, Salamanca, Valladolid et Zamora (GAYUBO, GonZALEZ \& TORRES, 2000 ; GAYUBO, NiEVES-ALDREY et al., 2004b; BAÑOS-PICON et al., 2007). Italie du nord, Toscane, Marches et Sicile (PAgLIANO \& NEGRISOlo, 2005), également en Calabre (DollFUSS, 2006). Trouvé aussi en Corse, Ghiarghia (commune de Zilia), 6.07.1993 (Y. Barbier leg.).

p. 142. GENRE ECTEMNIUS Dahlbom. Dans sa révision des espèces d'Asie et d'Océanie, LECLERCQ (1999) fournit une clé des sous-genres et groupes d'espèces de la faune mondiale, ainsi qu'une clé des espèces dans laquelle on trouve la plupart des espèces connues aussi d'Europe. JACOBS (2006) propose une nouvelle clé des espèces paléarctiques du sous-genre Thyreocerus.

p. 147. Ligne 4 du $\S 5$ de la clé des femelles d'Ectemnius, lire : fig. 41e (au lieu de 40e)

p. 150. A la fin du $\S 21$ ' de la clé des femelles, il faut ajouter le chiffre 22 , qui renvoie à l'alternative suivante.

p. 156. Ectemnius nigritarsus (Herrich-Schaeffer). Ligne 5 de la description de la femelle : deux petites taches aux tergites III et IV [au lieu de II-IV]. Espèce citée comme nouvelle pour la Haute-Autriche (GUSENLEITNER, 2000), déjà signalée de Basse-Autriche, Kärnten, Steiermark et Burgenland. Citée autrefois des environs de Poznam par Kohl, l'espèce n'a jamais été retrouvée en Pologne, où sa présence semble douteuse (WISNIOWSKI, 2004). Nouvelle pour la Lithuanie (BUDRYS, 2001). En France, sa présence dans les Alpes de Savoie a été signalée par LECLERCQ (1949), mais omise sur la carte 42 de la présente Faune. Nouveaux départements français : Aisne, près de Laon, 18.06.2005, 1 femelle (VAGO, 2005b). - Isère, Grenoble, 15.08.2003, 1 femelle (J. Smit leg.).

p. 157. Ectemnius cavifrons (Thomson). Nouvelle référence éthologique, sur la capture des proies et l'accouplement (JACOBI, 2001). Signalé comme nouveau pour le Vorarlberg, en Autriche (GuSENLEITNER, 1999) et pour l'Estonie (LuIG \& VoOlMA, 2000). Trouvé aussi en Bulgarie (JACOBS, 2005b ; LJUBOMIROV, 2006) et en Sicile (TOMARCHIO \& TURRISI, 2006). Nouveaux départements français : Aveyron, Cransac, 12.08.2008, 1 mâle (M. Aubert leg.). 
- Haut-Rhin, Colmar, Turckheim, 400 m, 10.07.2000, 1 mâle ; Munster, 1000 m, 5.07.1999, 1 femelle, et 900 m, 30.07.1999, 1 femelle (J. Smit leg.). - Vaucluse, Apt, Bonnieux, 400m, 10.07.2000, 1 mâle (J. Smit leg.).

p. 158. Ectemnius lapidarius (Panzer). Les diverses phases du comportement d'accouplement on été récemment décrites par THEUNERT (2007b). L'espèce est citée pour la première fois d'Estonie (Luig \& Voolma, 2000). Présente en Italie du nord, également en Toscane, Abruzzes et Sicile (PAgliano \& Nigrisolo, 2005). Aussi en Bulgarie (LJUbOMIROV, 2001, 2006 ; JACOBS, 2005b) et en Grèce : Thessalie (K. Standfuss leg.).

p. 161. Ectemnius ruficornis (Zetterstedt). Présent en Roumanie, Bulgarie et Ukraine (Dollfuss, 2004). Signalé récemment des Asturies, en Espagne (GAYUBO, GONZÁlEZ et al., 2006). Connu seulement du nord de l'Italie (PAgliano \& Negrisolo, 2005).

p. 162. Ectemnius sexcinctus (Fabricius). Cité de Bulgarie, notamment du sud, monts Rhodope (StoyanOV \& LJUbOMIROV, 1997 ; LJUbOMIROV, 2006). Nouveaux départements français : Gard, montagne de l'Espérou, au nord de Le Vigan, 1200 m, 21.08.1992, 1 mâle (J.-M. Maldès leg.). - Loire, Doizieux 31.07.46, 1 femelle (A. Moussa leg.). - Meurthe-etMoselle, Vandoeuvre-les-Nancy (G. Jacquemin leg.). - Vosges, Tendon (Y. Barbier leg.).

p. 162. Ectemnius borealis (Zetterstedt). De nouvelles captures en Hongrie (JóZAN, 1993), Bulgarie (LJUBOMIROV, 1999, 2006), Slovaquie (PADR \& LUKAS, 1994), République Tchèque (KULA \& TYRNER, 2003) et Slovénie (DOLLFUSS, 2004). A notre connaissance, aucune observation récente en Espagne. Nouveau département français : Lozère, Lou Galabert (Trelans) 21.07.1994 (Godeau \& Patiny leg.).

p. 164. Ectemnius dives (Lepeletier \& Brullé). Espèce citée récemment de Bulgarie (LuUbOMIROV, 1999, 2000, 2006 ; JACOBS, 2005b) et de deux localités du sud de la Grèce, d'après la capture de deux exemplaires en juin 1992 (GIACHINO et al., 2000).

p. 165. Ectemnius guttatus (Vander Linden). Nouveau pour la Bulgarie (LJUBOMIROV, 1999, 2006). Nouveau département français : Ardèche, Saint-Martial, lac, 830 m, 1 femelle 23.07.1994 (J. Hamon leg.).

p. 167. Ectemnius rugifer (Dahlbom). Cité de Rhodes (LECLERCQ, 1993) et de Bulgarie (Dollfuss, 2004). En Espagne, signalé des provinces de Salamanca, Badajoz, Madrid et Zamora (GAYUBO, GONZÁLEZ et al., 2002 ; GAYUBO, NIEVES-ALDREY et al., 2004b ; GAYUBO, GonZÁlez et al., 2006). En Italie, depuis le Nord jusque dans les Pouilles (PAGLIANO \& NEGRISOLO, 2005).

p. 168. Ectemnius confinis (Walker). Nouveau pour la Bulgarie (STOYANOV, 1996; JACOBS, 2005b) et pour la Pologne (WISNIOWSKI \& KOWALCZYK, 1998 ; WISNIOWSKI, 2004). Cité aussi de République Tchèque, Slovaquie, Slovénie, Hongrie, Roumanie et Grèce (DoLLFUSS, 2004 ; STANDFUSS \& STANDFUSS, 2006b). Trouvé en Italie du nord et du sud, en Sicile et en Sardaigne (PAGLIANO \& NeGRISOlO, 2005). Nouveau département français : Haut-Rhin, Colmar, canal Rhin, 1.08.2002, 1 mâle (J. Smit leg.).

p. 173. Ectemnius meridionalis (A. Costa). Concernant la présence de l'espèce en CharenteMaritime, Saint-Trojan-les-Bains, remplacer : JANVIER (1977b) par : Janvier (inédit, détermination de l'espèce confirmée par J. Leclercq).

p. 173. Ectemnius rubicola (Dufour \& Perris). Atteint l'Estonie au nord (LUIG \& VoolmA, 2000). Cité de Bulgarie (Dollfuss, 2004 ; LJUBOMIROV, 2006) et de Sicile (Negrisolo, 1995a ; PAGLIANO \& NEGRISLO, 2005).

p. 174. Ectemnius schlettereri (Kohl). Espèce nouvelle pour la Pologne : "Malopolska Upland" (cité par WISNIOWSKI, 2004). 
p. 175. Ectemnius cephalotes (Olivier). Signalé d'Estonie (LECLERCQ, 1993 ; LUIG \& Voolma, 2000). Repris en Espagne, dans les provinces de Soria, Madrid et Salamanca (GONZALEZ, GAYUBO et al., 2003 ; NIEVES-AldREY et al., 2003 ; GAYUBO, NIEVES-ALDREY et al., 2004a, b ; CRUZ-SANCHEZ et al., 2005). Présent dans de nombreuses régions d'Italie du nord, aussi des Abruzzes, Campanie, Basilicate, Sicile et Sardaigne (PAGLIANO \& PESARINI, 1995; PAGliano \& NegRisolo, 2005). Deux exemplaires trouvés en Grèce près de Ioánina le 20.06.1992 (GiAChino et al., 2000). Cité aussi de Bulgarie (Dollfuss, 2004 ; JACOBS, 2005b ; LJUBOMIROV, 2006).

p. 178. Ectemnius fossorius (Linné). Dans le paragraphe consacré aux synonymies, les références à deux sous-espèces asiatiques indiquent $c f$. Tsuneki $1977: 7$ et $1977: 9$. Il s'agit de Tsuneki, 1977c dans la liste ci-dessous (qui modifie celle donnée p. 293 dans la bibliographie du vol. 1).

- 1977a. Remplacer Ibidem par Special Publications of the Japan Hymenopterists Association, $\mathrm{n}^{\circ} 3: 1-27$.

- 1977b. (sans changement).

- 1977c. Taxonomic notes on some species of Crabronine wasps of Japan and Korea (Hymenoptera, Sphecidae). Ibidem, $\mathrm{n}^{\circ} 5: 1-9$.

Espèce cité d'Estonie (LUIG \& Voolma, 2000). Sa répartition en Espagne est indiquée sur la carte 30 donnée par TORMOS et al. (1994) qui figure trois points, dans les provinces de Cataluña, Castilla-La Mancha et Valencia. D'autres captures récentes dans les Asturies et dans la province de Zamora (GAYUBO, GONZÁLEZ et al., 2006; BAÑOS-PICON et al., 2007). En Italie, présent dans le Nord, aussi en Toscane, Ombrie et Sicile (PAgLiAno \& NEGRIsOlO, 2005). L'espèce serait menacée de disparition dans plusieurs régions d'Allemagne ; cependant des captures récentes ont été signalées à Berlin et dans le Brandebourg. Nouveaux départements français : Gironde, marais de Bruges, sur des ombelles d'Angelica (BASSET, 2001). - Haut-Rhin, Biesheim, 6.08.2007, 1 mâle ; 12.08.2007, 1 femelle; 12.08.2007, 1 femelle (M. Aubert leg.).

p. 179. Ectemnius kriechbaumeri (Kohl). Nouveaux départements français : Aude, Conques-sur-Orbiel, 8.06.2005, 1 mâle sur inflorescences de Pastinaca (J. Bitsch leg.). Isère, Grenoble, mont Rachais, 17.06.1992, 1 femelle (G. Manzoni).

p. 180. Troisième paragraphe : lire Croatie (au lieu de Craotie).

p. 180. Ectemnius lituratus (Panzer). A notre connaissance aucune capture récente en Espagne depuis la citation d'une localité de la province de Valence (GAYUBO \& TORMOS, 1984). Trouvé en Grèce (Dollfuss, 2004), en particulier en Thessalie, 1 mâle en août, pendant la période 1999-2005 (STANDFUSS \& STANDFUSS, 2006b). Nouveau département français : Sarthe : Cré, 2 mâles, 31.07 .1994 (L. Faillie leg.). En Gironde, BASSET (2001) dit que l'espèce est fréquente dans les marais de Bruges, près de Bordeaux, mais ne semble pas se trouver ailleurs dans le département.

p. 181. Ectemnius spinipes (A. Morawitz). Espèce très rare, retrouvée en Suisse, dans le Valais, 1 femelle, le 31.07.1999 (LiTT, 2004). En Autriche, 1 mâle le 23.07.2002, dans le "Burgenland", nouveau pour cette région (GUSENLEITNER, 2002) ; d'autres captures en 2003 dans le "Steiermark" (GuSENLEITNER, 2003). En Pologne, une capture récente dans le "Sandomierz Basin" (WISNIOWSKI, 2004). En Italie, seules les provinces du Trentino-Alto Adige et de Liguria sont mentionnées dans la Faune de Pagliano \& Negrisolo (2005). A notre connaissance, la dernière capture en France est une nouvelle localité de l'Isère, Mens, domaine de Raud, 665/723m, 31.07.1992, 1 femelle (L. Leseigneur leg.).

p. 183. Ectemnius crassicornis (Spinola). JACOBS (2005b, 2006) cite diverses localités de Croatie, Bulgarie et Grèce, ainsi que de Turquie et d'Uzbekistan. Chez les mâles d'Asie mineure, il distingue deux "formes" d'après la conformation de l'article 6 des antennes, avec des formes intermédiaires. 
p. 190. Entomognathus dentifer (Noskiewicz). Sa présence à Chypre, signalée dans la première édition de la présente Faune, résulte d'une confusion avec l'espèce E. libanonis (Kohl) qui existe à Chypre, alors que E. dentifer n'est pas connu de cette île (LECLERCQ, 1996). Nouveau département français : Vaucluse, Apt (R. Fonfria leg.).

p. 195. Lestica alata (Panzer). Citée de plusieurs provinces d'Italie : Veneto, TrentinoAlto Adige, Lombardia, Piemonte et Toscana (Pagliano \& Negrisolo, 2005). A notre connaissance aucune capture récente en France.

p. 196. Lestica subterranea (Fabricius). Capture de deux exemplaires au nord-est de la Grèce : Promahónas (Séres) le 15.06.1992 (GiACHINO et al., 2000). En Italie, trouvée dans le Nord : Veneto, Trentino-Alto Adige, Piemonte, Valle d'Aosta, aussi en Calabre et Sardaigne (PAgliano \& NigRisolo, 2005). En France, plusieurs exemplaires (11 femelles et 2 mâles) capturés dans le Haut-Rhin, Munster 900m, entre 1998 et 2005 (J. Smit leg.).

p. 198. Lestica clypeata (Schreber). JACOBI (2000) discute le rapport entre la morphologie particulière de l'avant-corps des mâles et le comportement d'accouplement. Signalé d'Estonie (Luig \& VoOlma, 2000). Nouveau département français : Indre, La Châtre, juillet 1986, 1 femelle (L. Boudet leg.).

p. 206. Lindenius albilabris (Fabricius). Première citation de Sicile : Armerina (SCHMIDEGGER, 2003). Nouveau département français : Loiret, Ardon (A. Larivière leg.).

p. 208. Lindenius laevis A. Costa. Première citation de Sicile : Agrigento (SCHMIDEGGER, 2003).

p. 208. Lindenius major Beaumont. Cité d'Italie du Nord : Liguria, sans précision de localité ni de date de capture (PAGLIANO \& NeGRISOlO, 2005).

p. 210. Lindenius melinopus (Kohl). Cité de deux provinces d'Italie : Liguria et Toscana (PAgLiano \& Negrisolo, 2005). Nouveau département français : Bouches-du-Rhône, Eygalières (R. Fonfria leg.).

p. 211. Lindenius mesopleuralis (F. Morawitz). Présent également en Sicile (PAGLIANO $\&$ NEGRISOLO, 2005). Sa répartition en Espagne est indiquée sur la carte 25 fournie par TORMOS et al. (1994). Une liste de Sphecidae de France communiquée par J.-Y. Cretin (Besançon) en date du 9.03.1993, comporte l'indication de 3 femelles et 6 mâles provenant de la Vendée, Barbastre, île de Noirmoutier, pris le 20.07.1969.

p. 212. Lindenius panzeri (Vander Linden). A la ligne 3 de la description du mâle, lire : "chez les mâles les plus grands" [au lieu de : chez les plus mâles les plus grands]. Présent en Italie du Nord, aussi en Toscane, Latium, Pouille et Sicile (PAgliano \& NigRIsOlO, 2005). Nouveaux départements français : Hautes-Alpes, Gap, 900 m, 16.08.2003, 1 femelle (J. Smit leg.). - Alpes-Maritimes, Casterine, vallée des Merveilles, 1550 m, 8.08.2004, 1 mâle (J. Smit leg.).

p. 213. Lindenius parkanensis Zavadil. Cité de Bulgarie, Roumanie, Kazakhstan et Turquie (DOLLFUSS, 2006).

p. 213. Lindenius peninsularis (Kohl). Cette espèce, non prise en compte dans la première édition de la Faune des Sphecidae d'Europe occidentale, est connue seulement d'Espagne : provinces de Avila, Caceres, Salamanca, Almeria et Murcia (GAYUBO, 1982b, 1984 ; LECLERCQ, 1989, 1993 ; DollfuSS, 2006). Elle n'a été trouvée ni en France ni au Portugal contrairement à ce qui est écrit dans la monographie des Crabroniens de LECLERCQ (1954). C'est une espèce proche de L. ibericus, qui s'en distingue notamment par les caractères suivants : collare bas, avec une faible échancrure médiane; sillon scapal sans pointe ou avec un minuscule tubercule arrondi ; angles latéraux du lobe médian du clypéus beaucoup plus aigus; dessus de la tête et du thorax à ponctuation très fine et dense, relativement mats ; aire pygidiale de la femelle à côtés presque droits, à ponctuation uniformément dense (BEAUMONT, 1956a ; LECLERCQ, 1989). 
p. 213. Lindenius pygmaeus (Rossi). A la ligne 3 du paragraphe décrivant les caractères de l'espèce, lire : "propodeum lisse, non striolé" [au lieu de : pronotum lisse, non striolé]. Nouveau département français pour la sous-espèce armatus : Puy-de-Dôme, Mirefleurs, 5.08.1994, 2 femelles (F. Durand leg.).

p. 215. Lindenius subaeneus Lepeletier \& Brullé. En France quelques exemplaires observés dans le département de l'Aisne, Chemizy-Ailles, le 28.06.2005 (VAGO, 2005b).

p. 215. GENRE RHOPALUM Stephens.

Dans sa clé des espèces américaines, LECLERCQ (2002) appelle deux espèces qui se trouvent aussi en Europe : $R$. clavipes (Linné) et coarctatum (Scopoli).

p. 220. Rhopalum beaumonti Moczar. SCHMIDT (1997) a précisé et figuré la forme du tubercule frontal et des articles basaux des antennes du mâle de cette rare espèce, trouvée en Allemagne, Autriche, Hongrie, Ukraine, Kazakhstan et Turquie.

p. 220. Rhopalum coarctatum (Scopoli). Nouvelle observation éthologique : selon une étiquette de collection, un exemplaire de cette espèce aurait été obtenu le 23.06.1972 à partir d'un fourreau de Psychide (Microlépidoptère) pris en Angleterre, dans le Surrey (UFFEN, 1996). Signalé en Estonie (Luig \& Voolma, 2000), en Bulgarie (LJubomirov, 2000, 2006), en Espagne provinces de Cantabria, Huesca et Salamanca (GAYUBO, GONZÁLEZ et al., 2006), dans plusieurs provinces du nord et du centre de l'Italie (PAGLIANO \& NEGRISOLO, 2005), cité récemment de Sicile (TOMARCHIO \& TURRISI, 2006). Nouveaux départements français : Gard, Vauvert (J. Leclercq leg.). - Haut-Rhin, Colmar, canal Rhin, 4.07.2001, 1 mâle (J. Smit leg.).

p. 222. Rhopalum gracile Wesmael. Plusieurs captures de cette espèce, sous le nom de $R$. nigrinum Kiesenwetter, faites dans les années 1980-1990 en Espagne : province d'Alicante (TORREGrosa et al., 1993) et province de Soria (GAYUBO, GARCiA et al., 1999). Cité de diverses provinces d'Italie du nord et du centre, aussi de Campania, Calabria et Sardaigne (Pagliano \& Negrisolo, 2005). Signalé d'Estonie (Luig \& Voolma, 2000). Nouveaux départements français : Aisne, Chivres-en-Laonnois (J. J. Bignon leg.). - Loiret, Ardon, juillet 1990, 1 mâle (A. Larivière leg.).

p. 224. Rhopalum austriacum (Kohl). Cité récemment de Bulgarie (LJUBOMIROV, 2001) et de Pologne (WISNIOWSKI, 2002, 2004 et 2005a).

p. 225. Rhopalum clavipes (Linné). En Espagne, pris au piège Malaise, dans la Sierra de Guadarrama (province de Madrid) au cours des années 1989-1992 (GAYUBO, NIEVESALDREY et al., 2004a). Italie du Nord et du Centre, jusqu'en Campanie (PAGLIANO \& NEGRISOLO, 2005), également signalé de l'île de Montecristo (GENERANI et al., 1998 ; CANOVAI et al., 2000), et cité pour la première fois de Sicile (SCHMID-EGGER, 2003 ; TOMARCHIO \& TURRISI, 2006). Cité aussi de Bulgarie (LJubomirov, 1999, 2006). Deux nouveaux départements français : Isère, le Fontanil, à $10 \mathrm{~km}$ au nord-ouest de Grenoble, plusieurs exemplaires des deux sexes éclos en mai 1979 à partir de vieux bois de lierre à moitié pourri (A. Moussa leg.) ; SaintLaurent-de-Beaumont, 1 mâle, 12.08.1993 (A. Moussa leg.). - Loire, Doizieux, 31.08.1947, 1 femelle (A. Moussa leg.).

p. 229. Tracheliodes curvitarsus (Herrich-Schaeffer). La présence de cette espèce en Allemagne reste douteuse, aucune capture récente n'ayant été signalée (SCHMIDT \& SCHMIDEGGER, 1997). ZETTEL et al., (2004) précisent quelques caractères distinctifs de T. curvitarsus et de $T$. varus, considérées comme espèces jumelles ; ces auteurs mentionnent de nouvelles captures en Basse-Autriche ( 3 femelles prises en juillet 2003) et signalent pour la première fois sa présence en Bulgarie (captures de 1982, 1997 et 2003) et en République Tchèque (captures de 1973? et 1986) ; aussi en Grèce. ZETTEL et al., (2004) résument ce qui est connu de la biologie de l'espèce, en se référant notamment au travail de NEMKOV (1988). 
p. 231. Tracheliodes varus (Panzer). ZETTEL et al., (2004) confiorment l'interprétation donnée dans le vol. 1 de la Faune d'Europe occidentale (BITSCH \& LECLERCQ, 1993), qui considère $T$. varus comme une espèces distincte, mais très voisine, de T. curvistarsus. La conclusion de Zettel et al. résulte de l'examen de spécimens trouvés récemment en BasseAutriche, et aussi pour la première fois en Bulgarie, Slovaquie et République Tchèque. Les deux espèces, curvitarsus et varus, se trouvent dans les mêmes localités et chassent la même espèce de Fourmi, Liometopum microcephalum. Comme cette espèce de Fourmi n'existe pas en Corse, on doit supposer que les femelles de T. varus de Corse chassent une autre espèce de Fourmi.

\section{p. 233. Tribu des OXYBELINI.}

Le vol. 1 de la présente Faune a pris en compte deux genres d'Oxybelini représentés en Europe occidentale : Belomicrus et Oxybelus. Un troisième genre, Belomicroides, a été brièvement mentionné car cité d'Ukraine et de l'île de Rhodes. Une révision de la tribu a été entreprise ces dernières années par ANTROPOV. Dans un article de 2001, l'auteur russe a subdivisé le genre Belomicroides Kohl, 1899, en deux genres distincts : Belomicroides s. str. (espèce-type : Belomicroides schmiedeknechti Kohl, 1899) exclusivement africain, et Pseudomicroides (espèce-type : Belomicroides olgae Kazenas, 1979) surtout paléarctique. Sept espèces, précédemment attribuées au genre Belomicroides ont alors été transférées dans le nouveau genre Pseudomicroides. Dans un travail ultérieur, ANTROPOV (2005) a réhabilité le genre Oxybelomorpha Brauns, 1897, in KoHL (1897) que KoHL (1923) et les auteurs qui l'ont suivi avaient considéré comme synonyme de Belomicrus; il a transféré dans le genre Oxybelomorpha quatre espèces attribuées jusque là au genre Belomicrus.

\section{p. 234. GENRE BELOMICRUS A. Costa.}

Compte tenu des transferts indiqués ci-dessus, on recense actuellement cinq espèces de Belomicrus s. str. présentes en Europe. Quatre d'entre elles (B. antennalis, borealis, italicus et odontophorus) ont déjà été mentionnées dans le vol. 1 de la présente Faune. L'espèce $B$. steckii Kohl a été transférée dans le genre Oxybelomorpha. Une nouvelle espèce, Belomicrus gaetensis Gayubo, Asis \& Tormos, 1998, a été décrite d'après une femelle capturée le 27.05.1986 sur Sonchus tenerrimus L. dans le sud de l'Espagne, province d'Almeria. En outre l'article de GAYUBO, ASIS \& TORMOS, 1998, comporte une revue des espèces de Belomicrus trouvées dans la péninsule Ibérique.

p. 236. Belomicrus italicus A. Costa. L'espèce, connue de plusieurs pays d'Europe centrale et de la région méditerranéenne (Espagne, Portugal, Chypre), a été trouvée pour la première fois en Sicile, en 1999 et 2002, et à Malte en 1999 (SCHMID-EGGER, 2003). Nouvelle espèce pour la Bulgarie, d'après plusieurs captures en 1997 et 1998 (LJUBOMIROV, 1999).

\section{p. 237. GENRE OXYBELOMORPHA Brauns, 1897 (Espèce-type : Oxybelomorpha} kohlii Brauns, in Kohl, 1897).

Une seule espèce connue d'Europe occidentale : Oxybelomorpha steckii (Kohl, 1923) = Belomicrus steckii Kohl, 1923, et auteurs ultérieurs jusqu'à ANTROPOV, 2006. D'abord citée du sud de la France, sans localité précise, l'espèce n'a jamais été retrouvée dans ce pays. Différentes captures récentes faites dans le centre et le sud de l'Espagne, aussi au Portugal et au Maroc.

\section{GENRE PSEUDOMICROIDES Antropov, 2001}

L'espèce initialement décrite sous le nom de Belomicroides fergusoni par BEAUMONT (1960) à partir d'un exemplaire mâle de l'île de Rhodes, a été rattachée au genre Pseudomicroides Antropov, 2001. Une femelle et un mâle de cette espèce ont été capturés en juin et juillet 1996 dans le sud de la Grèce (Péloponnèse), ce qui confirme sa présence en Europe (ARENS, 2002). 


\section{p. 237. GENRE $\boldsymbol{O X Y B E L U S}$ Latreille}

Clé des espèces européennes du genre (GUICHARD, 1993) et clé des espèces d'Italie (PAgliano \& NegRisolo, 2005). L'examen des nombreux exemplaires d'Oxybelus Latreille et de Brimocelus Arnold conservés dans les collections du "Biologiezentrum" de Linz, Autriche, ont permis à DoLLFuSs (2008) d'établir l'inventaire détaillé des espèces et de préciser leur localisation dans la région paléarctique et dans le reste du Monde.

p. 240. Point 12 de la clé des femelles, ligne 2 du $2^{\mathrm{e}}$ paragraphe, lire : "une grande tache au-dessous des fémurs 1 et 2" [et non des fémurs 2 et 3].

p. 246. Oxybelus andulasiacus Spinola. Dans la présente Faune, le taxon andalusiacus a été retenu comme sous-espèce de $O$. lamellatus (p. 255). Le taxon andalusiacus, qui semble être le seul présent en Europe, est souvent considéré comme une espèce distincte, notamment par des auteurs actuels qui confirment sa présence dans différentes provinces d'Espagne (GAYUbo \& Tormos, 1984 ; GAYUBO \& Heras, 1986 ; GAYUBO, AsIS \& Tormos, 1998 ; TORREGROSA et al., 1993), au Portugal et en Italie, y compris la Sardaigne et l'île de Lampedusa (Pagliano, 1990 ; Negrisolo 1995a ; Pagliano \& SCARAMOZzino, 1995 ; Pagliano \& NEGRISOLO, 2005).

p. 246. Oxybelus argentatus Curtis. Une note brève sur la nidification et les proies d'après des observations faites en Lombardie (MARTINOLI et al., 1996) et une liste des proies en Angleterre (ARCHER, 1999b). La sous-espèce gerstaeckeri P. Verhoeff (= beaumonti Verhoeff) a été signalée de Bavière (WICKL \& WICKL, 1994 ; BLÖSCH, 1996), d'Italie du nord (NEGRISOLO, 1995a), aussi de la province de Soria en Espagne (GAYUBO, GARCIA et al., 1999).

p. 249. Oxybelus bipunctatus Olivier. Nouvelles observations sur la nidification de l'espèce dans l'Etat de New York (KURCZEWSKI, 2002). Nouveaux départements français : Aisne, Rocourt-Saint-Martin, 21.06.2002, 1 femelle ; Courtemont-Varennes, 12.06.2003, 1 femelle (E. Gros leg.). - Hautes-Alpes, Gap, rivière Durance, 600 m, 19.08.2003, 1 femelle ; Réotier, 875 m, 26.08.2003, 4 femelles (J. Smit leg.). - Haut-Rhin : Geiswasser, 25.07.08, 1 femelle ; Kunheim, 26.07.2008, 1 femelle (M. Aubert leg.).

p. 251. Oxybelus dissectus Dahlbom. GuICHARD (1993) fait remarquer que les taches claires, généralement blanches sur les exemplaires de France, sont jaunes sur des exemplaires de Corse et de Grèce. La dernière capture de cette espèce, dans le Land de Baden-Württemberg, remonte à 1936 (BURGER et al., 1998). Première citation pour la Bulgarie, d'après la capture d'un mâle à Melnik le 24.08.1990 (JACOBS, 2005b). Nouveaux départements français : Puyde-Dôme, Neschers (F. Durand leg.). - Haute-Savoie, embouchure de la Dranse (Thononles-Bains) (DREWES, 2003).

p. 252. Oxybelus dusmeti Mingo Pérez. D'après Guichard (1993), qui a examiné les cotypes de cette espèce, le mâle décrit ne diffère pas de $O$. mucronatus ; ce n'est vraisemblablement pas le mâle de l'espèce, qui reste inconnu. Selon Dollfuss (2008), les mâles de $O$. dusmeti provenant d'Espagne et présents dans les collections du "Biologiezentrum" de Linz ont les mandibules brunâtres, et les mésopleures avec une rugosité verticale caractéristique, semblable à celle des femelles.

p. 253-254. Oxybelus haemorrhoidalis Olivier. Observations sur le comportement de nidification de la sous-espèce haemorrhoidalis dans les Alpes italiennes (Lombardie) en 1995; les nids sont approvisionnés de Diptères Sarcophagides (NicOli ALDINI, 2004). La carte 97 (p. 250) de la présente Faune est à compléter pour trois département français : Ain, Sermayer, dunes, piège jaune, 21.06.1995, 3 femelles ; parmi celles-ci, deux peuvent être attribuées à la sous-espèce victor, la troisième femelle est plus ou moins intermédiaire entre les sous-espèces haemorrhoidalis et victor (J. Hamon leg.). - Loiret, Ardon, 20.07.1989, 1 mâle de la sous-espèce haemorrhoidalis (A. Larivière leg.). - Vaucluse, Carpentras et Orange, 
plusieurs exemplaires sous le nom de $O$. victor victor dans la collection J. de Beaumont, Lausanne (inventaire J. Hamon).

p. 255. Oxybelus lamellatus Olivier. Dans le vol. 1 de la présente Faune, l'espèce $O$. lamellatus a été interprétée comme comportant deux sous-espèces, lamellatus lamellatus et lamellatus andalusiacus. PULAWSKI (2006) résume les caractéristiques d'O. lamellatus, en suivant l'interprétation de BEAUMONT (1950). Comme indiqué ci-dessus (complément de la p. 246), plusieurs auteurs actuels considèrent andalusiacus comme une espèce distincte, présente notamment en Espagne, Baléares, Italie, Sardaigne et Afrique du Nord. La larve et le comportement nidificateur de $O$. lamellatus, sans précision de sous-espèce, ont été étudiés par AsIS et al. (1997) et par TORMOS et al. (2000) d'après des observations faites près du rivage méditerranéen dans la province de Valencia, Espagne. Les nids, comportant une seule galerie oblique terminée par une cellule, forment des agrégations denses dans un sol sablonneux ; les proies sont des Diptères Brachycères (35 espèces recensées) ; il existe différents parasitoïdes (Diptères Sarcophagidae et Hyménoptères Chrysididae). Le taxon lamellatus s. str. se trouve notamment en Afrique du Nord, à Chypre, Rhodes et en Turquie (LECLERCQ, 1993).

p. 256. Oxybelus latidens Gerstaecker. Comme FAESTER en 1949, GUICHARD (1993) considère $O$. latidens comme étant seulement une forme de l'espèce $O$. subspinosus, cette dernière ayant une coloration et une ponctuation variable. Mais PULAWSKI, dans son catalogue (2007), maintient l'espèce $O$. latidens. DOLLFUSS (2008), qui a vu de nombreux spécimens intermédiaires entre les deux taxa, pense que la synonymie est justifiée, mais il n'a pas examiné les types.

p. 256. Oxybelus latro Olivier. Espèce rare en Allemagne, signalée de plusieurs Länder (Baden-Württemberg, Bayern, Rheinland-Westfalen, Hessen, Sachsen et Brandebourg), mais le plus souvent par des captures qui datent des années 1940 (SCHMIDT \& SCHMID-EGGER, 1997). Signalée comme très en danger ou disparue dans le Brandebourg (WITT, 1996 ; BURGER et al., 1998 ; JACOBS, 2000). En Autriche, citée du Burgenland, mais sans date de capture (DoLLFUSS et al., 1998). Quelques captures en Espagne, dans les provinces de Soria et de Tarragona (GAYUBO, GARCIA et al., 1999 ; DreWES, 2003), aussi en Croatie et Bulgarie (LJUBOMIROV, 2000). Cité de différentes provinces d'Italie, du nord au sud, dont la Sicile (PAGLIANO \& NEGRISOLO, 2005).

p. 257. Oxybelus lineatus (Fabricius). Captures plus ou moins récentes en France : Gironde, Lacanau-Océan, 21.07.1990, 1 femelle (M. Willemse leg.). - Landes, Capbreton, 3.08.86, 2 mâles (J.-Cl. Teulou leg.).

p. 258. Oxybelus mandibularis Dahlbom. Cité, ces dernières années, de Grande-Bretagne, de nombreux Länder d'Allemagne, d'Autriche, Pologne, Bulgarie et Hongrie. En Espagne, GAYUBO \& SANZA (1986) rapportent les captures, en 1985, d'une femelle et de deux mâles, respectivement dans les provinces de Burgos et de Soira, captures qui viennent s'ajouter à celle, dejà mentionnée, de la province de Zamora. Dans la Faune d'Italie, PAGLIANO \& NEGRISOLO (2005) signalent cette espèce du Piémont et de Sardaigne. Nouveaux départements français : Manche, Beaubigny (Y. Barbier leg.). - Puy-de-Dôme, Orléat, juillet 1995, 1 femelle, 2 mâles ( $F$. Durand leg.).

p. 258. Oxybelus mucronatus (Fabricius). Très peu de captures récentes en Allemagne (CÖLLN \& JAKUBZIK, 2008). En Bulgarie capture de 2 mâles, l'un en 1982, l'autre en 1997 (JACOBS, 2005b). Commun au Luxembourg, où 41 exemplaires ont été capturés entre 2000 et 2004 (FEITZ et al., 2006). Nouveaux départements français : Ardèche, Chauzon, sur Mentha, 22.08.1995, 1 femelle (J. Hamon leg.) ; Pradons, 23.07.1995, 2 mâles (J. Hamon leg.). Corse, Bucchinera (C. Wonville leg.) et PAgliano \& NEgRisolo, 2005. - Lot-et-Garonne, Nérac, août 1980 (J. C. Teulou leg.). - Haut-Rhin, Munster, 15.07.1998, 1 mâle (J. Smit leg.). 
p. 260. Oxybelus occitanicus Marquet. LJUBOMIROV (2000) le cite de Bulgarie. L'espèce ne figure pas dans la récente Faune des Sphecidae d'Italie (PAgliano \& Negrisolo, 2005).

p. 260. Oxybelus quattuordecimnotatus Jurine. Nouveaux départements français : Ain, Sermoyer, dunes, piège jaune, 21.06.1994, 4 mâles, et 31.07.1995, 1 femelle (J. Hamon leg.). - Hautes-Alpes, Embrun, 26.08.2003, 1 femelle, 1 mâle (J. Smit leg.) ; Isère, Arandon, 300 m, 23.07.2004, 1 femelle et 1 mâle (J. Smit leg.).

p. 261. Oxybelus spectabilis Gerstaecker. Signalé de nombreuses provinces d'Espagne. En Afrique du Nord, présent aussi en Tunisie (H. \& M. Tussac leg.).

p. 262. Oxybelus subspinosus Klug. Nouveau département français : Alpes-de-HauteProvence, Moustiers-Sainte-Marie, 700 m, 18.07.2001, 1 femelle (J. Smit leg.).

p. 263. Oxybelus trispinosus (Fabricius). Observations sur le comportement de nidification et sur les larves : NICOLI ALDINI (2004). Signalé pour la première fois du Luxembourg (FEITZ et al., 2003). Nouveau département français : Ardèche, Astet, col du Pendu, 1380 m, 25.07.1994, 1 femelle, 1 mâle (J. Hamon leg.).

p. 264. Oxybelus uniglumis (Liné). Nouveau pour la Bulgarie (LJUBOMIROV, 2001, 2006). Nouveaux départements français : Aisne, Chézy-sur-Marne, bac jaune, 23.06.1991, 1 femelle (E. Gros leg.). - Alpes-de-Haute-Provence, Moustiers-Sainte-Marie, 700 m, 18.07.2001, 1 femelle (J. Smit leg.). - Ardèche, Chauzon sur Mentha 22.07.1995, 1 mâle (J. Hamon leg.) ; Saint-Thomé (R. Wahis leg.). - Haute-Loire, Tence (Muséum de Grenoble, H. Maneval leg.).

p. 265. Oxybelus variegatus Wesmael. Observations sur le comportement de nidification et les proies : NicOLI ALDINI (2004). Nouveaux départements français : Ardèche, Saint-Thomé (R. Wahis leg.). - Isère, Saint-Laurent-de-Beaumont, piège jaune, 29.07.1990, 1 femelle (A. Moussa leg.).

p. 293. Modifier les références de TsUNAKI, 1977, conformément à la liste donnée plus haut, p. 178, à propos d'Ectemnius fossorius.

p. 322, ligne 7. Lire : 4. ingricus (au lieu de ingrinus).

\section{AUTEURS CITÉS}

La liste ci-dessous donne le détail de toutes les références bibliographiques citées dans les "Compléments au volume 1 de la Faune des Sphecidae d'Europe occidentale", sauf celles déjà données dans le volume 1 publié en 1993. Cette liste a été mise à jour en décembre 2008.

ABENIUS J. \& LARSSON K., 2006. - First record of Crossocerus exiguus (Hymenoptera: Crabronidae) from Sweden. Entomologisk Tidskrift, 127 : 151-152.

AISTLEITNER E., 2004. - Fragmenta entomofaunistica VIII. Weitere Daten zur HautsanguinelüglerFauna von Vorarlberg, Austria occ. und dem Fürstentum Liechtenstein. Nachritenblatt der Bayerischen Entomologen, $53: 58-62$.

AMiet F. \& MoretTi M., 2002. - Neue und interessante Bienen- und Wespenarten für das Tessin und die Schweiz von Waldbrandgebieten (Hymenoptera: Aculeata: Apidae, Pompilidae, Sphecidae). Mitteilungen der Schweizerischen Entomologischen Gesellschaft, 75 : 173-182.

ANTROPOV A.V., 2001. - Pseudomicroides, a new genus of digger wasps of the tribe Oxybelini (Hymenoptera, Sphecidae, Crabroninae). Revision of the world fauna. Zoologischesky Zhurnal, 80 : 809-830. 2005. - Revision of digger wasps of the genus Oxybelomorpha (Hymenoptera, Crabronidae, Oxybelini). A. The O. kohlii species-group. Entomological Review, 85 : 504-516.

— 2006. - Revision of digger wasps of the genus Oxybelomorpha (Hymenoptera, Crabronidae, Oxybelini). 6. Species groups "persa" and "steckii". Zoologischesky Zhurnal, 85 : 193-208 [en russe]. ARCHER M.E., 1993. - The aculeate wasps and bees (Hymenoptera: Aculeata) at Duncombe Park in Watsonian Yorkshire. Naturalist, 118 : 37-44.

1994. - Recent rare and scare wasps and bees (Hym., Aculeata) recorded from Guernesey and Herm. Entomologist's Monthly Magazine, 130 : 103-104. 
1995. - Aculeate wasps and bees (Hymenoptera: Aculeata) of Blaxton common in Watsonian Yorkshire with the introduction of a new national quality scoring system. Naturalist, 120 : 21-29.

- 1996a. - The aculeate wasps and bees (Hymenoptera: Aculeata) of Hemm and Sark, Channel Islands. Entomologist's Gazette, 47 : 53-59.

1996b. - The aculeate wasps and bees (Hym., Aculeata) of Sherwood forest in Nottinghamshire and the development from a national quality assessment scheme. Entomologist's Monthly Magazine, 132 : 35-44.

1996c. - The aculeate wasps and bees (Hymenoptera: Aculeata) of Shipley Glen in Watsonian Yorkshire with a "then" and "now" comparison. Naturalist, 121 : 53-59.

— 1997. - The aculeate wasps and bees (Hymenoptera: Aculeata) of two calcareous localities in Watsonian Yorkshire: Burton Leonard lime quarries and cave wold. Naturalist, 122 : 45-52.

- 1999a. - The aculeate wasps and bees (Hymenoptera: Aculeata) of the Ainsdale-Formby sand dunes of the Lancashire coast compared with other nerthern sites. British Journal of Entomology and Natural History, 12 : 1-10.

1999b. - Prey of Oxybelus argentatus Curtis (Hym., Sphecidae). Entomologist's Monthly Magazine, $135: 106$.

2000a. - The sphecid wasps, Crabro and Crossocerus (Hymenoptera: Sphecidae) of Watsonian Yorkshire. Naturalist, $125:$ 45-60.

2000b. - The aculeate wasps and bees (Hymenoptera: Aculeata) of Saltfleetby-Theddlethorpe NNR in Watsonian Lincolnshire, including statistical procedures for estimating species richness. Entomologist's Gazette, 51 : 107-115.

2001. - The wasps and bees (Hymenoptera: Aculeata) of the river bank at Beninbrough and Keswick

Fitts in Watsonian Yorkshire. Entomologist's Gazette, 52 : 105-115

2002. - The Wasps, Ants and Bees (Hymenoptera: Aculeata) of Watsonian Yorkshire. Yorkshire Naturalist's Union, Weymouthm, Dorset, 200 pp.

— 2003a. - The wasps and bees (Hymenoptera: Aculeata) of remnant sites in intensive agricultural countryside in Watsonian Yorkshire: Brayton Barff, Ringhay Wood, South Cliff Common and Thornton Ellers. Naturalist, 128 : 49-59.

2003b. - The wasps and bees (Hymenoptera: Aculeata) of Messingham sand quarry in Watsonian Lincolnshire with special reference to resident and tourist species. Naturalist, 128 : 93-102.

2004a. - The wasps and bees (Hym., Aculeata) of Devils Spittleful Nature Reserve in Watsonian Worcestershire. Entomologist's Monthly Magazine, 140 : 39-49.

2004b. - Recorder's eighth report of the aculeate Hymenoptera in Watsonian Yorkshire. Naturalist, 129 : $75-76$.

2004c. - The wasps and bees (Hymenoptera: Aculeata) of York's Victorian cemetery in Watsonian Yorkshire. Naturalist, 129 : 145-153.

2005a. - The wasps and bees (Hymenoptera: Aculeata) of three more remnant sites in Watsonian Yorkshire: Haw Park, Stutton and Fulford Ings, York. Naturalist, 130 : 81-92.

2005b. - The wasps and bees (Hymenoptera: Aculeata) of English heritage sites in Watsonian Yorkshire. Entomologist's Monthly Magazine, 141 : 21-31.

2006a. - The wasps and bees (Hym., Aculeata) of Rauceby Warren Nature Reserve in Watsonian Lincolnshire with a comparison with other Lincolnshire sites. Entomologist's Monthly Magazine,

$142: 11-22$.

2006b. - The wasps and bees (Hymenoptera: Aculeata) of Pollington Quarry in Watsonian Yorkshire. Naturalist, $131:$ 67-76.

2006c. - The wasps and bees (Hym., Aculeata) of the open sandy habits of Highgate Common in Watsonian Staffordshire. Entomologist's Monthly Magazine, 142 : 207-218.

2007a. - The wasps and bees (Hymenoptera: Aculeata) of Thetford Warren Lodge in Watsonian West Norfolk. Entomologist's Monthly Magazine, 143 : 141-151.

2007b. - Solitary aculeate wasps and bees (Hym.) recorded from the Isle of Portland (Dorset) during early July 2006. Entomologist's Monthly Magazine, 143 : 151.

2007c. - Solitary aculeate wasps and bees (Hym.) recorded from the South Devon coast during 2006. Entomologist's Monthly Magazine, 143: 152.

2007d. - Interesting aculeate wasp and bee (Hym.) records from Watsonian Yorkshine, 2006.

Entomologist's Monthly Magazine, 143 : 152. 
2007e. - Current knowledge of British Aculeate Hymenoptera with special reference to the occurrence of high quality species on priority habitats. British Journal of Entomology and Natural History, 20 : 75-94.

2008a. - Revisiting the solitary wasps and bees (Hymenoptera: Aculeata) of Burton Leonard Lime quarries and Duncombe Park in Watsonian Yorkshire. Naturalist, 133 : 21-27.

2008b. - The wasps and bees (Hymenoptera: Aculeata) of the sand dunes of Bamburgh, Northumbria and Sandscale Haws, Culbria. Entomologist's Monthly Magazine, 144 : 131-144.

ARENS W., 2002. - Das Weibchen von Pseudomicroides fergusoni (de Beaumont) (Hymenoptera, Sphecidae, Crabronidae), zugleich Erstnachweis der Gattung in Europa. Linzer biologische Beiträge, 34 : 513-519.

ARENS W. \& HARTMANN P., 2004. - Faltenwespen, Wegwespen, Goldwespen und andere Aculeate Hymenopteren in der Umbebung von Bayreuth. Berichte naturwissenschaftiche Gesellschaft Bayreuth, $25: 267-277$.

ASIS J.D., TORMOS J. \& GAYUBO S.F., 1997. - Descripcion de la larva madura de Oxybelus lamellatus Olivier y O. spectabilis Gerstaecker (Hymenoptera, Sphecidae). Miscellània Zoológica, 20 : 59-64.

BAÑOS-Picón L., GAYUBO S.F., ASIS J.D., TORMOS J. \& GONZÁLEZ J.A., 2007. - Diversidad de la comunidad de avispas spheciformes de una zona agricola abondonada del oeste español (Hymenoptera: Apoidea: Ampulicidae, Sphecidae y Crabronidae). Nouvelle Revue d'Entomologie, (n. s.), 23 [2006] : 249-266.

BASSET G., 2001. - Contribution à la connaissance des Sphecidae (Hymenoptera) de Gironde. Bulletin de la Société Linnéenne de Bordeaux, 29 : 75-85.

BEAVIS I. C., 2000. - Aculeate Hymenoptera on the Isles of Scilly. Entomologist's Gazette, 51 : 59-68.

— 2001. - Aculeate Hymenoptera of Tunbridge Wells and the Central High Weald. Entomologist's Gazette, 52 : 97-129.

-2005. - Aculeata Hymenoptera on the Isles of Scilly - First supplement. Entomologist's Gazette, 56 : 189-204.

BITSCH J., BARBIER Y., GAYUBO S. F., SCHMIDT K. \& OHL M., 1997. - Hyménoptères Sphecidae d'Europe occidentale. Vol. 2. Faune de France, 82 : 1-429. Fédération française des Sociétés de Sciences Naturelles, Paris.

Bitsch J., Dollfuss H., BouceK Z., Schmidt K., Schmid-EgGer C., GAyubo S. F., Antropov A. V. \& BARBIER Y., 2001. - Hyménoptères Sphecidae d'Europe occidentale. Vol. 3. Faune de France, 86 : 1-459. Fédération française des Sociétés de Sciences Naturelles, Paris.

BITSCH J. \& LECLERCQ J., 1993. - Hyménoptères Sphecidae d'Europe occidentale. Vol. 1. Généralités Crabroninae. Faune de France, 79 : 1-325. Fédération française des Sociétés de Sciences naturelles, Paris.

BLÖSCH M., 1996. - Beitrag zur Kenntnis der Grabwespenfauna (Hym., Sphecidae) des neuen Naturschutzgebietes "Tennenlohner Forst" bei Erlangen. Galathea, 12: 2-8.

1998. - Nachtrag zur Grabwespenfauna (Hym., Sphecidae) des Naturschutzgebietes "Tennenloher Forst" bei Erlangen.Galathea, 14 : 165-169.

- 2000. - Die Grabwespen Deutschlands. Lebensweise, Verhalten, Verbreitung. In Die Tierwelt Deutschlands, 71 Teil, Hymenoptera II, 480 pp. Deutsches Entomologisches Institut, Eberswalde.

2003. - Die Besiedlung von morschen Zaunpfählen durch Stechimmen im Jahresverlauf. Galathea, 19 : $77-86$.

BOLTON B., 1995. - A new general catalogue of the ants of the world. Harvard University Press, Cambridge, Massachussets.

BRothers D. J., 1999. - Phylogeny and evolution of wasps, ants and bees (Hymenoptera, Chrysidoidea, Vespoidea and Apoidea). Zoologica Scripta, 28 : 233-249.

BUDRYS E., 2001. - Five new for Lithuanian fauna species of Sphecidae and Crabronidae (Hymenoptera, Apoidea). Acta Zoologica Lituanica, 11 : 388-390.

Burger F., SAure C. \& OeHLKe J., 1998. - Rote Liste und Artenliste der Grabwespen und weiterer Hautflüglergruppen des Landes Brandenburg (Hymenoptera: Sphecidae, Vespoidea part, Evanoidea, Trigonalyoidea). Naturschutz und Landschaftspflege in Brandenburg, 7 : 24-43 (Beilage Heft 2).

CANOVAi R., GiANNOTTI P. et al., 2000. - Biodiversità: Compilazione delle species dell'entomofauna e dei piccoli vertebrati della Corsica e delle Toscana marittima. Unione Europa. Progetto interreg II. Toscana-Corsica, 1997-1999. L'attività scientifica delle Università di Pisa e Corte, p. 75-86.

CARTIER G., 2000. - Addenda aux insectes de Rueil-Malmaison. Déterminations postérieures au 14 septembre 1999. L'Entomologiste, 56 : 161-165. 
CElary W., 1998. - Hymenoptera Aculeata (excluding Formicoidea) of Babia Góra Mt. and adjacent area. Acta Zoologica Cracoviensia, 41 : 207-225.

CÖLLN K., ESSER J. \& JAKUBZIK A., 2003. - Stechimmen (Hymenoptera Aculeata) in Abgrabungen und Brüchen des Nordwestens von Rheinland-Pflaz. Dendrocopos, 30 : 49-66.

CöLln K. \& JAKUBZIK A., 1999. - Hymenoptera Aculeata der Keuper-Scharren südwestlich der Hungerburg (Mutillidae, Myrmosidae, Sapygidae, Tiphiidae, Pompilidae, Sphecidae et Apidae). Fauna und Flora in Rheinland-Pfalz, $9: 21-45$.

— 2000. - Nachträge zum Arteninventar einzelner Tiergruppen in Gönnersdorf (Landkreis Daun/eifel). Dendrocopos, 27 : 225-230.

— 2008. - Die Grabwespe Oxybelus mucronatus (Fabricius, 1793), ein Neufund für Rheinland-Pfalz. Bembix, $26: 8-10$.

CRETIN J.-Y., 1999. - Liste commentée des Hyménoptères récoltés en 1998 par la technique des pièges colorés sur le territoire de la Réserve Naturelle de la Massane. Office pour l'information écoentomologique du Languedoc-Roussillon, Laboratoire Arago Banyuls-sur-Mer, 9 pp.

CRUZ-SÁnChEZ M. A., GAYUBO S. F., GonZÁlez J. A. \& TORRES F., 2005. - La comunidad de avispas Spheciformes de un melojar del oeste español: diversidad y dinámica temporal (Hymenoptera, Apoidea, Ampulicidae, Sphecidae y Crabronidae). Nouvelle Revue d'Entomologie (n.s.), 21 : 213-238.

Cungs J. \& JAKUBZIK A., 2001. - Seltene Weg- und Grabwespen (Hymenoptera Aculeata: Pompilidae et Sphecidae) Luxemburgs, mit besonderem Bezug auf das ehemalige Erzabbaugebiet Haardt bei Düdelingen. Bulletin de la Société des Naturalistes du Luxembourg, 101 : 117-128.

DATHE H. H. \& BLANK M., 2004. - Nachträge zum Verzeichnis der Hautflügler Deutschlands, Entomofauna Germanica Band 4 (Hymenoptera). (1). Entomologische Nachrichten und Berichte, 48 : 179-184.

DENTON J. S., 1998. - Recent records of scarcer aculeate Hymenoptera in North Hampshire and Surrey. Entomologist's Monthly Magazine, 134 : 350.

DOLLFUSS H., 1994. - Rote Liste gefährdeter Grabwespen (Hymenoptera, Sphecidae), pp. 95-104. In J. Gepp (ed.) Rote Listen gefärdeter Tiere Oesterreichs. Grüne Reihe des Bundesministeriums für Umwelt, Jugend und Familie, vol. 2. Styria Medien Service, Graz. 355 pp.

- 2004. - The Crabroninae wasps of "Biologiezentrum Linz" - collection in Linz, Austria (Hymenoptera, Apoidea, Crabronidae). Part 1. Linzer biologische Beiträge, 36 : 761-784.

2006. - The Crabronidae wasps of "Biologiezentrum Linz" - Collection in Linz, Austria (Hymenoptera, Apoidae, Crabronidae), Part 2. Linzer biologische Beiträge, 38 : 505-532.

2008. - The crabronid wasps of the genus Oxybelus Latreille 1796 and Brimocelus Arnold 1927 of "Biologiezentrum Linz" collectionnin Linz, Austria (Hymenoptera, Apoidea, Crabronidae). Linzer biologische Beiträge, 40 : 463-505.

DOLlFuss H., GuSENLEITNER J. \& BREGANT E., 1998. - Grabwespen im Burgenland (Hymenoptera: Sphecidae). Stapfia, 55 : 507-552.

DREWES B., 1998. - Zur Besiedlung einer Kiesgrube im Landkreis Stade durch Grabwespen, Wildbienen und weitere aculeate Hymenopteren (Hymenoptera: Aculeata). Drosera, 1998 : 45-68.

— 2003. - Revision der Grabwespen der Sammlung des Landesmuseums für Natur und Mensch Oldenburg (Hymenoptera, Sphecidae). Drosera, 2003 : 131-144.

DUNK K. V. D. \& AMON F. J., 2002. - Untersuchungen zur Insektenfauna auf der Stromleitung-Sandstrasse Schwaig bei Nürnberg. Galathea, 18 : 145-163.

ELLIGSEN H., 1998. - Die Stechimmenfauna von Magerrassen und Uferabbrüchen in der ukranischen Vorkarpaten (Hymenoptera: Aculeata). Entomologische Zeitschrift, 108 : 65-79.

ERHARDT H., 1999. - Die Stechimmenfauna einer stillgelegten Tonkuhle im Landkreis Ammerland (Hymenoptera: Aculeata). Drosera, 1999 : 69-94.

ESSER J. \& CÖLLN K., 2002. - Bedeutung von Tuff- und Lavagruben für die Stechimmenfauna (Hymenoptera: Aculeata) der Eifel. Fauna und Flora in Rheinland-Pfalz, 9 : 1115-1154.

ESSER J. \& JAKUBZIK A., 2003. - Wildbienen und Wespen (Hymenoptera: Aculeata) aus dem Niederrheinischen Tiefland in den Sammlungen des zoologischen Instituts der Universität zu Köln. Decheniana, $156: 287-296$.

ESSER J., JAKUBZIK A. \& SONNENBURG G. H., 2004a. - Stechimmen (Hymenoptera: Aculeata) in NordrheinWestfalen: Aenderungen gegenüber dem Verzeichnis der Hautflügler Deutschlands. Bembix, 18 : 13-23.

Esser J., JAKUBZIK A., SONNEnburg G. H. \& WoydaK H., 2004b. - V. Artlisten der Stechimmen Nordrhein-Westfalen, p. 255-270. In Cölln K. et al. (eds.) Stechimmen in Nordrhein-Westfalen. 
Schriftenreihe der Landesanstalt für Ökologie, Bodenordnung und Forsten Nordrhein-Westfalen, 20 : $1-327$.

FEITZ F., GOLDEN R., MELCHIOR E. \& SchNEIDER N., 2006. - Wespen und Wildbienen des Naturschutzgebiets "Baggerweieren" im "Haff Réimech", Luxembourg (Insecta, Hymenoptera, Aculeata). Bulletin de la Société des Naturalistes luxembourgeois, 106 : 75-99.

FEITZ F., SCHNEIDER N. \& PAUly A., 2001. - Hyménoptères Aculéates nouveaux ou intéressants pour la faune luxembourgeoise (Hymenoptera, Aculeata). Bulletin de la Société des Naturalistes luxembourgeois, 100 : 129-146.

— 2003. - Hyménoptères Apocrites nouveaux ou intéressants pour la faune luxembourgeoise (Hymenoptera, Apocrita). Bulletin de la Société des Naturalistes luxembourgeois, 104 : 79-88.

FELTON J. C. \& SCHNEIDER N., 1994. - Matériaux pour un catalogue des Hyménoptères du Luxembourg. Bulletin de la Société des Naturalistes luxembourgeois, 95 : 287-294.

FLÜGEL H.-J., 2003. - Wespenfunde am Lebendigen Bienenmuseum Knüllwald (Hymenoptera Aculeata et Gasteruptiidae). Philippia, $11: 1-16$.

FREUNDT R. \& ILLMER J., 2003. - Einige bemerkenswerte Funde von Hautflüglern (Hymenoptera) im Kreis Wesel/Niederrein. Bembix, $17: 8-13$.

GAYUBo S. F., AsIS J. D. \& ToRmos J., 1990. - The genus Belomicrus A. Costa, 1871 in the Iberian Peninsula (Hymenoptera, Sphecidae, Crabroninae. Mitteilungen der münchner entomologischen Gesellschaft, 88 : 13-18.

GAYUBO S. F. \& BORSATO W., 1994. - Contribución al conocimiento de la esfecidofauna italiana. Bollettino del Museo Civico di Storia naturale di Verona, 18 [1991] : 195-225.

GAyubo S. F., GARCía J., TORRES F. \& GonZÁlez J., 1999. - Contribución al conocimiento de los esfécidos (Hymenoptera, Sphecidae) de la provincia de Soria (España). Boletín de la Real Sociedad española de Historia natural, Sección Biologica, 95 : 87-99.

Gayubo S. F., GonZÁlez J. A., Nuez A. DE lA, Asis J. D. \& Tormos J., 2006. - Especies nuevas o interesantes de Spheciformes para Europa y la Península Ibérica (Hymenoptera: Ampulicidae, Sphecidae y Crabronidae). Boletin de la Sociedad entomológica aragonesa, 39 : 205-212.

Gayubo S. F., GonzÁlez J. A., TORMos J. \& AsIS J. D., 2002. - Especies nuevas o interesantes de esfeciformes para la Península Ibérica (Hymenoptera: Ampulicidae, Sphecidae y Crabronidae). Boletin de la Sociedad entomológica aragonesa, 31 : 83-92.

— 2008. - Diversidad de avispas Sphceciformes en el Parque Natural de Las Batuecas — Sierra de Francia (Salamanca, España) (Hynemnoptera : Ampulicidae, Sphecidae y Crabronidae). Nouvelle Revue d'Entomologie (n.s.), 24 : 131-147.

GAYUBO S. F., GONZÁLEZ J. A. \& TORRES F., 2000. Estudio de una comunidad de esfécidos en la zona natural de "Las Arribes del Duero" (Salamanca, Oeste Español) (Hymenoptera, Sphecidae). Fragmenta Entomologica, 32 : 181-209.

Gayubo S. F., Nieves-Aldrey J. L., GonzÁlez J. A., Asis J. D., Rey del CASTillo C. \& Tormos J., 2004a. - La communidad de avisas esfeciformes de la estación biogeológica de El Ventorillo (Sector Medio de la Sierra de Guadarrama Madrid, España) (Hymenoptera, Apoidea, Ampulicidae, Sphecidae y Crabronidae). Nouvelle Revue d'Entomologie (n.s.), 21 : 125-156.

Gayubo S. F., Nieves-Aldrey J. L., GonzÁlez J. A., Tormos J., Rey del CASTillo C. \& Asis J. D., 2004b. - Diversidad de avispas spheciformes (Hymenoptera, Apoidea, Ampulicidae, Sphecidae y Crabronidae) colectas mediante trampa Malaise en el Monte Pardo (Madrid, España). Boletín de la Real Sociedad española de Historia natural, Sección Biologica, 99 : 105-113.

GAYUBO S. F., TORMOS J. \& ASIS J. D., 1993. - New or little known sphecid wasps for the Iberian Peninsula. Bollettino de la Società entomologica italiana, 124 : 201-208.

Generani M., Pagliano G., ScAramozzino P. L. \& Strumia F., 1998. - Nuovi imenotteri dell'isola di Montecristo (Arcipelago Toscano) (Hymenoptera: Tenthredinidae, Gasteruptioniidae, Evaniidae, Ichneumonidae, Chrysididae, Tiphiidae, Scoliidae, Formicidae, Sphecidae, Apoidea). Frustula Entomologica, n.s., 21 : 75-83.

2003. - Gli Imenotteri delle isole di Capraia, Giglio, Gorgona, Pianosa e Montecristo (Arcipelago Toscano) (Insecta: Hymenoptera). Frustula Entomologica, 24 [2001] : 1-74.

Georgiev G. \& LJubomirov T., 2000. - Species of Sphecidae (Hymenoptera) reared from swellings of Saperda populnea (L.) (Coleoptera: Cerambycidae) in Bulgaria. Acta Zoologica Bulgarica, $52: 41-44$. 
Georgiev G., Ljubomirov T., Raikova M., IVAnOV K. \& SAKAlian V., 2004. - Insects inhabitants of old larval galleries of Saperda populnea (L.) (Coleoptera: Cerambycidae) in Bulgaria. Journal of Pest Science, 77 : 235-243.

Giachino P. M., Grosso F., Marchetti M., Pagliano G., Scaramozzino P. L. \& Vailati D., 2000. - Elenco degli Hymenoptera Leucospididae (Chalcidoidea) e Aculeata raccolti in Grecia nel 1992. Bollettino del Museo regionale di Scienze naturali di Torino, 17 : 87-130.

GonzÁlez J. A., GAYubo S. F., Asis J. D., ToRmos J. \& GARCiA C., 2003. - Estructura y dinámica temporal de la comunidad de avispas esfeciformes (Hymenoptera: Apoidea: Ampulicidae, Sphecidae y Crabronidae) en un pastizal de la Cuenca Alta del Duero (España). Boletin de la Sociedad entomológica aragonesa, $32: 59-65$.

GONZÁLEZ J. M., GAYUBO S. F. \& TORRES F., 1998. - Diversidad y abundancia de esfécidos de la Submeseta Norte (España). Boletín de la Real Sociedad española de Historia natural, Sección Biologica, 94 : 71-85.

— 1999. - Estudio comparativo de la biodiversidad de esfécidos colectados mediante trampa Malaise en un sector arenoso de la cuenca del Duero (España) (Hymenoptera, Sphecidae). Nouvelle Revue d'Entomologie (n.s.), 15 [1998] : 351-370.

- 2000. - Diversidad y abundancia da esfécidos en una zona pirenaica con influencia mediterranea. Nouvelle Revue d'Entomologie (n.s.), 17 : 13-33.

GOROBCHISHIN V., 1993. - [Sphecidae of Kanev Nature Reserve, Ukraine], p. 46-47. In M.G. Chornyi (ed.), Pidsumki 70-richnoi diyalnosti Kanivs'kogo Zapovenika ta perspaektivi rozvitku zapovidnoi spravi v Ukraini. Kaniv, 189 p.

— 1995. - [Digger wasps (Hymenoptera, Sphecidae) of Kanev Reserve and bordering territories]. Izvestiya Kar'kovskogo Entomologicheskogo Obshchestva, 3 : 17-19.

- 1996. - [Biotopic distribution of sphecid wasps in Kanev Nature Reserve and adjacent territories, Ukraine]. Zapovidna Sprava v Ukraïni, 2 : 52-53.

GUICHARD K. M., 1993. - European Oxybelus with a note on Oxybelus dusmeti Perez, 1966 (Hymenoptera, Sphecidae). Entomofauna, 14 : 529-536.

GuSENLEITNER J., 1995. - Hymenopterologische Notizen aus Oesterreich - 3 (Insecta: Hymenoptera aculeata). Linzer biologische Beiträge, 27 : 159-167.

1996a. - Hymenopterologische Notizen aus Oesterreich - 4 (Insecta: Hymenoptera aculeata). Linzer biologische Beiträge, 28 : 5-13.

1996b. - Hymenopterologische Notizen aus Oesterreich - 6 (Insecta: Hymenoptera aculeata). Linzer biologische Beiträge, 28 : 809-816.

— 1998. - Hymenopterologische Notizen aus Oesterreich - 9 (Insecta: Hymenoptera aculeata). Linzer biologische Beiträge, 30 : 498-501.

1999. - Hymenopterologische Notizen aus Oesterreich - 12 (Insecta, Hymenoptera aculeata). Linzer biologische Beiträge, 31 : 593-601.

2000. - Hymenopterologische Notizen aus Oesterreich - 13 (Insecta: Hymenoptera aculeata). Linzer biologische Beiträge, 32 : 953-962.

2002. - Hymenopterologische Notizen aus Oesterreich - 15 (Insecta: Hymenoptera aculeata). Linzer biologische Beiträge, 34 : 1123-1126.

— 2003. - Hymenopterologische Notizen aus Oesterreich - 17 (Insecta: Hymenoptera aculeata). Linzer biologische Beiträge, 35 : 863-868.

— 2005. - Hymenopterologische Notizen aus Oesterreich - 19 (Insecta: Hymenoptera aculeata). Linzer biologische Beiträge, 37 : 1203-1205.

HAESELER V., 1997. - Veränderung der Artenspectren der auf den Ostfriesischen Inselnistenden solitären Wespen und Bienen - eine Folge anthropogener Einwirkungen. Schriftenreihe Nationpalpark niedersächsisches Wattenmeer, 2 : 28-35.

-2001. - Zur Wespen- und Bienenfauna des Brookdeichs bei Oldenburg i.O. (Hymenoptera: Aculeata). Oldenburger Jahrbuch 2001, 101 : 257-286.

— 2003. - Ameisen, Wespen und Bienen der Weserinsel Harriersand bei Bremen. - Oldenburger Jahrbuch 2001, 103 : 333-363.

— 2005. - Stechimmen der Steller Heide bei Bremen im Zeitraum 1985 bis 2004 (Hymenoptera: Aculeata). Abhandlungen des naturwissenschaftlichen Vereins zu Bremen, 45 : 621-656.

HAESELER V. \& RITZAU C., 1998. - Zur Aussagekraft wirbelloser Tiere in Umwelt - und Naturschutzgutachten - was wird tätsachtlich erfasst? Zeitschrift für Ökologie und Naturschutz, 7 : 45-66. 
HARVEY P. R., 1996. - Some interesting Hymenoptera records for 1995. Essex Naturalist (n.s.), 13 : 23-28. HARVEY P. R. \& PlanT C. W., 1996. - A provisional list of the bees, wasps and ants (Hymenoptera) of Essex. Essex Naturalist (n.s.), 13 : 43-115.

HEIDE V.D. A. \& METSCHER H., 2003. - Zur Bienen- und Wespenbesiedlung von Taldünen der Ems und anderen Trockenstandorten im Emsland (Hymenoptera: Aculeata). Drosera, 2003 : 95-130.

HERRMANN M., 1999. - Einfluss von Flächengrösse und Isolation auf die Präsenz von Grabwespen (Hymenoptera: Sphecidae). Drosera, 1999 : 1-22.

IVANOV K. \& LJUBOMIROV T., 2001. - Hymenoptera (Insecta) in Kresna Gorge (SW Bulgaria). In P. Beron (ed.) Biodiversity of Kresna Gorge (SW Bulgaria), p. 205-215 [en bulgare, résumé en anglais].

JACOBI B., 2000. - Paarungsverhalten und Anatomie von Lestica-Arten (Hym., Sphec., Crabroninae). Verhandlungen des westdschen Entomologen Tag, 1999 : 139-146.

2001. - Beutfang und Paarungsverhalten bei Ectemnius (Clytochrysus) cavifrons (Thomson, 1870) (Hymenoptera, Sphecidae, Crabroninae). Verhandlungen des westdschen Entomologen Tag, 2000 : 81-88.

JACOBS H.-J., 1999. - Zur Unterscheidung kleiner Weibchen von Crossocerus podagricus (Vander Linden, 1829) und C. congener (Dahlbom, 1844) (Hym., Sphecidae). Bembix, 12 : 26-27.

2000. - Rote Liste der gefährdeten Grabwespen Mecklenburg-Vorpommerns (Hymenoptera Aculeata:

Sphecidae). Umweltministerium Mecklenburg-Vorpommern, p. 1-20.

2005a. - Ergänzungen zur Grabwespenfauna Deutschlands (Hym., Crabronidae). Entomologische Nachrichten und Berichte, 49 : 149-150.

— 2005b. Lindenius anatolicus Beaumont 1967 - Erstnachweis in Europa und weitere Angaben zur Grabwespenfauna Bulgariens (Hymenoptera: Sphecidae, Crabronidae). Linzer biologische Beiträge, $37: 435-456$.

2006. - Beschreibung von E. zonsteini sp. n. und Bestimmungstabelle der palärktischen Ectemnius (Thyreocerus)-Arten. Beiträge zur Entomologie, 56 : 105-113.

— 2007. - Die Grabwespen Deutschlands. Ampulicidae, Sphecidae, Crabronidae. Bestimmungsschlüssel. In Die Tierwelt Deutschlands 79. Teil, 207 p. Goecke \& Evers, Keltern.

JAKUBZIK A., 1996. - Weg- und Grabwespen von Köln (Hymenoptera, Aculeata: Pompilidae und Sphecidae). Decheniana, Beihefte, 35 : 241-272.

JAKUBZIK A. \& CÖLLN K., 1993. - Zu den Brombeerstengel bewohnenden Hymenopteren (Hymenoptera, Aculeata) von Wehlen (Kreis Bernkastel-Wittlich). Dendrocopos, 20 : 33-141.

— 1996. - Weg- und Grabwespen (Hymenoptera, Aculeata: Pompilidae et Sphecidae) des Nordwestens von Rheinland-Pfalz. Fauna Flora Rheinland-Pfalz, 8 : 391-420.

— 2005. - Beitrag zur Kenntnis der Stechimmen (Hymenoptera, Aculeata) des Bausenbergs bei Niederzissen. Dendrocops, 32 : 61-66.

JAKUBZIK A., SCHLÜTER R. \& CÖLLN K., 1998. - Weg- und Grabwespen (Hymenoptera, Aculeata: Pompilidae et Sphecidae) des Nordwestens von Rheinland-Pfalz. I. Nachtrag Fauna und Flora in Rheinland-Pfalz, 8 : 1173-1193.

JóZAN Z., 1993. - The Scoliidea and Sphecoidea fauna of the Bükk National Park (Hymenoptera), p. 411421. In Mahunka S. (ed.), The fauna of the Bükk National Park. Vol. I. Magyar Természettudományi Múzeum, Budapest. 456 pp.

— 1995. - [Beiträge zur Kenntnis der Aculeatenfauna (Hymenoptera) Nationalparkes Duna-Dráva (Ungarn, Süd-Transdanubien)]. Dunántúli Dolgozatok Természettudományi Sorozat, 8 : 99-115.

JuNK C., SCHOSS F. \& ScHOSS R., 1994. - Flora und Fauna der Steingrube "Schoofsboesch" bei Bettendorf. Bulletin de la Société des Naturalistes luxembourgeois, 95 : 49-102.

KAZENAS V.L., 2001. - [Fauna and biology of sphecid wasps (Hymenoptera, Sphecidae) of Kazakhstan and Central Asia]. Kazgos INTI, Almaty, 333 pp.

KLEIN W., 1996. - Die Graafwespen von de Benelux. Hymenoptera, Sphecidae. Jeugdbondsuitgeverij, Utrecht, $130 \mathrm{pp}$.

KOPONEN M., 1994. - [New distribution records and comments on Crossocerus in Finland]. Sahlbergia, $1: 21-22$.

KOWALCZYK J. K., 1994. - [Aculeata (Hymenoptera) of Lagiewniki forest in Lódz]. Acta Universitatis Lodziensis. Folia Zoologica, 2: 65-91.

KOWALCZYK J. K. \& KRZEPTOWSKI M., 1995. - [New records of aculeate wasps from Tatra National Park and adjacent areas, Poland]. Wiadomosci Entomologiczne, 14 : 188-189. 
KowAlCZYK J. K., SzCZEPKo K. \& SwiATEK M., 2002. - [Sphecid wasps (Hymenoptera: Sphecidae) of surroundings of the Fiel Study Centre of the Lódz University in Kampinoski National Park]. Wiadomosci Entomologiczne, 20 : 147-156.

KRAATZ O., 2005. - Grabwespen und Bienen eines militärisch genutzten Binnendünengeländes im Nordwesten Niedersachsens (Hymenoptera: Sphecidae, Apidae). Drosera, 2005 : 97-126.

KraUS M. \& Floren A., 2002. - Pflanzwespen (Hymenoptera, Symphyta) und Stechimmen (Chrysididae, Pompilidae, Sphecidae) aus Baumkronenbenebelungen (Fogging) von Eichen und Rotbucjen in Bayern (Unterfranken), Thüringen (Hainich), Slovenien und Rumänien. Galathea, Suppl. 11 : 93-102.

KuHLMAnN M., 1993. - Kritisches Verzeichnis ausgewählter Stechimmenfamilien Westfalens (Hym., Aculeata). I. Chrysididae, Tiphiidae, Mutillidae, Sapygidae, Pompilidae, Eumenidae, Sphecidae und Apidae (excl. Apinae). Mitteilungen der Arbeitsgemeinschaft ostwestfälisch-lippischer Entomologen, 9:69-85.

2000. Die Struktur von Stechimmenzönosen (Hymenoptera Aculeata) ausgewählter Kalkmagerrasen des Diemeltales unter besonder Berücksichtigung der Nutzungsgeschichte und des Requisitienangebotes. Abhandlungen aus dem Westfälischen Museum für Naturkunde, 62 :3-102.

KUHLMANN M. \& QUEST M., 2003. - Stechimmenzönosen von Moorstandorten und eines Bruchwaldes sowie Ergebnisse einer dreijährigen Daueruntersuchung auf einer isolierten Lichtung in Nationalpark Bayerischer Wald (Hymenoptera, Aculeata). Nachrichtenblatt der bayerischen Entomologen, 52 : 46-59.

KUHLMANN M., WOLF H. \& WOYDAK H., 1999. - Rote Liste der gefährdeten Stechimmen (Wildbienen und Wespen, Hymenoptera Aculeata) Westfalens. 1. Fassung, pp. 563-574. In R. Wolff-Straub \& U. Wasner (eds.) Rote Liste der gefährdeten Pflanzen und Tiere in Nordrhein-Westfalen. Schriftenreihe der Landesanstalt für Ökologie, Bodenordnung und Forsten Nordrhein-Westfalen, 17 : 1-644.

KULA E. \& TYRNER P., 2003. - Hymenoptera (Aculeata) of spruce stands in the air-pollution region of Northern Bohemia. Journal of Forest Science, 49 : 200-207.

KURCZEWSKI F. E., 2002. - Seasonal variation in nesting behavior of Oxybelus bipunctatus (Hymenoptera: Sphecidae). Northeastern Naturalist, 9 (4) : 419-432.

LECLERCQ J., 1949. - Notes détachées sur les Hyménoptères aculéates de Belgique (4-8). Bulletin et Annales de la Société entomologique de Belgique, 85 : 180-183.

1993. - Hyménoptères Sphécides Crabroniens d'Europe et du Bassin Méditerranéen. Notes fauniques de Gembloux, 26 : 9-54.

— 1996. - Pour les Hyménoptères Sphécides Crabroniens paléarctiques du genre Entomognathus Dahlbom. Bulletin et Annales de la Société royale belge d'Entomologie, 132 : 261-264.

— 1999. - Hyménoptères Sphécidés Crabroniens du genre Ectemnius Dahlbom, 1845. Espèces d'Asie et d'Océanie, et groupes d'espèces de la faune mondiale. Notes fauniques de Gembloux, 36 : 3-83.

2000. - Hyménoptères Sphécides Crabroniens des Amériques du genre Crossocerus Lepeletier \& Brullé, 1835. Notes fauniques de Gembloux, 40 : 3-75.

- 2002. - Hyménoptères Crabronides Crabroniens des Amériques du genre Rhopalum Stephens, 1829. Notes fauniques de Gembloux, 48 : 3-115.

2007. - Hyménoptères Crabroniens de Grèce du genre Crossocerus Lepeletier \& Brullé 1835 (Hymenoptera : Crabronidae Crabroninae). Notes fauniques de Gembloux, 60 : 189-193.

2009. - Hyménoptères Crabroniens d'Asie du genre Crossocerus Lepeletier \& Brullé 1835 (Hymenoptera : Crabronidae Crabroninae). Faunistic Entomology - Entomologie faunistique, 61 (4) [2008] : 157-192.

LECLERCQ J. \& BARBIER Y., 1993. - Atlas de répartition des Crabroniens de France et des régions limitrophes (Hymenoptera, Sphecidae, Crabronini). Notes fauniques de Gembloux, 27 : 3-94.

LEFEBER V., 1998. - Bijen en Wespen (Hymenoptera Aculeata) in de Enci-Groeve van de Sint-Pietersburg bij Maastricht. Natuurhistorisch Maandblad, 87 : 174-186.

LiTT R., 2002. - Observations sur les Sphécides en Belgique (Hymenoptera: Sphecidae). Lambillionea, $102: 161-167$.

2004. - Les Hyménoptères du Valais (Suisse). Lambillionea, 104 : 216-222.

LJubomirov T., 1999. - Preliminary studies on the digger wasp fauna (Insecta: Hymenoptera: Sphecidae) in Vitosha Mountain. Acta Zoologica Bulgarica, 51 : 43-59.

2000. - Revised ckeck-list of digger wasps (Hymenoptera: Sphecidae) from the collection of N. Nedelkov at the National Museum of Natural History in Sofia. Historia naturalis bulgarica, 12 : 5-15. 
2001. - Further investigations on the digger wasp fauna (Insecta: Hymenoptera: Sphecidae) in the Vitosha Mountain (Bulgaria). Acta Zoologica Bulgarica, 53 : 45-59.

— 2006. - A survey of the faunistic investigations on the Hymenopteran families Siricidae, [...], Sphecidae, and Crabronidae from the Western Rhodopes - Bulgaria and Greece (Insecta Hymenoptera). In Beron P. (ed.) Biodiversity of Bulgaria. 3. Biodiversity of Western Rhodopes (Bulgaria and Greece). I. Pensoft \& Nat. Mus. Natur. Hist., Sofia, p. 524-545.

LJUBOMIROV T. \& BAN-CALEFARIU C. 2008. - Faunistic records of Crabronidae (Hymenoptera) from Maramures (Romania). Travaux du Muséum national d'Histoire naturelle "Grigpore Antipa", 60 : 201-207.

LOHRMANN V., OHL M., BLEIDORN C. \& PODSiAdlOWSKi L., 2006. - Molekulare Phylogenie der Apoidea (Hymenoptera) unter besonderer Berücksichtigung der Grabwespen ("Sphecidae"). Beiträge der Hymenopterologen-Tagung Stuttgart, 2006 : 26-28.

LUCHETTI D., 1993. - Liste preliminare degli Imenotteri dell'arcipelago della Maddalena (Sardegna). Bollettino de la Societa entomologica italiana, 125 : 103-108.

LUIG J. \& VoOLMA K., 2000. - [Arboreal digger wasps in Estonia II: subfamily Crabroninae (Hymenoptera, Sphecidae).] Metsanduslikud Uurimused, 32 : 121-128.

LUKAS J. \& LISKA P., 2005. - First records of digger wasps (Hymenoptera, Sphecidae) from Slovakia. Biologia, 60 : 550.

MAGDAlOU J.-A., 2006a. - Inventaire des Hyménoptères Sphecidae (Hymenoptera, Sphecidae). Travaux de la Réserve Naturelle de la Massane, 75, 18 p. Laboratoire Arago, Banyuls-sur-Mer.

— 2006b. - Contribution à la connaissance des Hyménoptères aculéates de la Réserve Naturelle du Mas-Larrieu (commune d'Argelès-sur-Mer, Département des Pyrénées-Orientales). Famille des Sphecidae. Réserve Naturelle du Mas-Larrieu, document, 24 p.

MANDERY K., 2005. - Bienen und Wespen (Hymenoptera: Apocrita) im Hainberg einem aussergewöhnlichen Sandlebensraum an der Rednitz, Gde. Oberasbach (Lkr. Fürth) u. Stadt Nürnberg. Galathea, 21 : 167-186.

Martinoli A., Casiraghi M., Passerini E., Bosco T. \& Andrietti F., 1996. - Nesting and nest's provisioning in Oxybelus argentatus (Hymenoptera : Sphecidae) in Alta Valtellina (Northern Italy). Proceedings of the XXth International Congress of Entomology, Firenze, p. 382.

MAUSS V. \& SCHINDlER M., 1998. - Nachweise von Bienen und Wespen im Kreis Nordfriesland, Schleswig-Holstein (Chrysididae, Apidae, "Sphecidae", Pompilidae, Vespidae). Bombus, 3 : 134-136.

MAUSS S., SCHRÖDER S., \& BOTTA C., 2000. - Untersuchungen zur Höhenverbreitung von Hummeln und sozialen Faltenwespen in Arbergebiet des bayerischen Waldes mit Anmerkungen zum Vorkommen solitärer Stechimmenarten (Hymenoptera: "Sphecidae", Apidae, Pompilidae, Vespidae). Nachrichtenblatt der bayerischen Entomologen, 49 : 71-79.

Melo G. A. R., 1999. - Phylogenetic relationships and classification of the major lineages of Apoidea (Hymenoptera), with emphasis on the crabronid wasps. Scientific Papers - University of Kansas, Natural history Museum, $14: 1-55$.

Mochi A. \& LuCHETTI D., 1993. - Note faunistiche su alcuni sfecidi italiani (Hymenoptera). Bollettino dell' Associazione romana di Entomologia, 47 [1992] : 103-107.

Negrisolo E., 1995a. - Hymenoptera Sphecidae. In Minelli et al. (eds.) Checklist delle specie della fauna italiana, 105 : 1-12. Edizioni Calderini Bologna.

1995b. - The aculeate communities (Hymenoptera Aculeata) of two coastal areas of the Veneto Region (north eastern Italy). Lavori della Società veneziana di Scienze naturali, 20 : 15-25.

NegRisolo E. \& PAGLIANO G., 1993. - Su alcuni Sphecidae catturati in Sardegna (Hymenoptera). Bollettino della Società Sarda di Scienze Naturali, 29 : 89-96.

NemKov P. G., 1988. - [Review of Holarctic digger wasps of the genus Tracheliodes A. Morawitz (Hymenoptera, Sphecidae, Crabroninae) with a description of two new species from the Soviet Far East]. Trudy Vsesoyuznogo Entomologischeskogo Pbshchesteva [horae Societatis Entomologicae Unionis Sovieticae], 70 : 116-125 [en russe].

2004. - Contribution to the knowledge of the species of Crossocerus Lepeletier et Brullé (Hymenoptera: Crabronidae, Crabroninae) described by K. Tsuneki. Proceedings of the Russain Entomological Society, 75 : 263-269.

NiCOLI ALDINI R., 2004. - Behavioural observations on three species of Oxybelus (Hymenoptera Sphecidae) nesting in syntopy. Redia, $87: 253-256$. 
Nieves-Aldrey J. L., Fontal-CaZalla F., Garrido-Torres A. M. \& Rey del Castillo C., 2003. - Inventario de Hymenoptera (Hexapoda) en El Ventorillo: un rico enclave de biodiversidad en la Sierra de Guadarrama (España central). Graellsia, 59 : 25-43.

NILSSON G. E., 1992. - [New records of Hymenoptera Aculeata from Sweden]. Entomologisk Tidskrift, 113 : 53-57 [en suédois].

OHL M., 2001. - Sphecidae, p. 137-143. In H. H. Dathe, A. Taeger \& S. M. Blank (eds.) Entomofauna Germanica, Band 4. Verzeichnis der Hautflügler Deutschlands. Entomologische Nachrichten und Berichte, Beiheft 7 : 1-178.

- 2003. - Kommentierter Katalog der Grabwespen Deutschlands (Hymenoptera: Sphecidae). Internet version der dedruckten Entomofauna Germanica. http://amor.cms.hu-berlin.de/ h0662dgt/akteg.html

— 2007. - Räuber, Parasiten, Kuckuncke. Die Evolution von Grabwespen und ihrer vielfältigen Beutefang- und Nestbaustrategie. Humboldt-Spektrum, 14 (Heft 2) : 44-48.

OHL M. \& BLEIDORN C., 2006. - The phylogenetic position of the enigmatic wasp family Heterogynaidae based on molecular data, with description of a new, nocturnal species (Hymenoptera: Apoidea). Systematic Entomology, $31: 321-337$.

OHL M. \& ENGEL M. S., 2007. - Die Fossilgeschichte der Bienen und ihrer nächsten Verwandten (Hymenoptera: Apoidea). Denisia, 20 : 687-700.

PADR Z. \& LUKÁS J., 1994. Stachelhautflügler aus dem Gebiet des Hohen Tatragebirges in der slovakischen Republik (Hymenoptera-Aculeata). Linzer biologische Beiträge, 26 : 887-904.

PAGLIANO G., 2003. - Ricerche Imenotterologiche nelle Isole di Lampedusa e Pantelleria (Hymenoptera Apocrita). Il Naturalista siciliano, S. IV, $27: 115-149$.

Pagliano G. \& Negrisolo E., 2005. - Hymenoptera Sphecidae. Fauna d'Italia, 40 : I-IX, 1-559.

PAGLIANO G. \& PESARINI F., 1995. - Nota preliminare sugli imenotteri sfecidi della Provincia di Ferrara (Hymenoptera, Sphecidae). Quaderni della Statione di Ecologia del civico Museo di Storia naturale di Ferrara, 8 : 81-95.

PAGLiAnO G. \& SCARAmOzZino P. L., 1995. - Hymenoptera Gasteruptionidae e Aculeata (esclusi Chrysidoidea, Mutillidae e Formicidae). In Massa B. (ed.) Arthropoda di Lampedusa, Linosa e Pantelleria (Canale di Sicilia, Mar Mediterranes). Il Naturalista siciliano, 19 (suppl.) : 723-738.

PAPP J. \& JÓZAN Z., 1995. - The dispersion and phenology of sawflies and aculeate wasps in the Sikfökút oak forest, Hungary (Hymenoptera). Folia Entomologica Hungarica, 56 : 133-152.

PAuly A., 1999. - Catalogue des Hyménoptères Aculéates de Belgique. Bulletin de la Société royale belge d'Entomologie, 135 : 98-125.

PAWLIKOWSKI T. \& KRUSZYNSKI T., 1996. - [The materials to studies on the community structure of Hymenoptera Aculeata in Poland. 7. Sphecid wasps (Hymenoptera, Sphecidae) in forest environments of the Torun Basin]. Acta Universitatis Nicolai Copernici, Biologia LI, 96 : 83-92.

Peeters T. M. J., ACHTERberg C. von, Heitmans W. R. B. et al., 2004. - De wespen en mieren van Nederland (Hymenoptera: Aculeata). Nederlandse Fauna, 6, 507 pp. Leiden.

PetiT J., 1997. - La Frayère de Lanaye (Province de Liège, Belgique). Colonisation d'un milieu neuf par les Hyménoptères Aculéates (Hymenoptera, Aculeata). Lambillionea, 97 : 343-354.

- 1998. - Fleurs et insectes. Les visiteurs de la renouée du Japon : Polygonum cuspidatum. Lambillionea, 98 : $106-117$.

PIPER R., 2007. - Nest building and prey stocking in Crossocerus megacephalus (Rossius) (Hym.: Sphecidae). The Entomologist's Record and Journal of Variation, 119 : 58.

PRENTICE M. A., 1998. - The comparative morphology and phylogeny of apoid wasps (Hymenoptera: Apoidea). Dissertation Thesis. University of California, Berkeley, 1439 p.

PRICE A., 2001. - The digger-wasp Crabro peltarius at Locko Park, Derbys. The Derbyshire \& Nottinghamshire Entomological Society Journal, 145 : 9.

PULAWSKI W. J., 2006. - Nomenclatural changes in Old World Crabronidae (Hymenoptera) with taxonomic comments and new distribution records. Journal of Hymenoptera Research, 15 : 164-170.

— 2007. - Bibliography of Sphecidae sensu lato. - Catalog of Genera and Species of Sphecidae sensu lato. - Family group names and classification. http://www.calacademy.org/research/entomology...

QUEST M. \& KUHLMANN M., 2005. - Stechimmenzönosen von Borkenkäferlücken in Nationalpark Bayerischer Wald. Nachrichtenblatt der bayerischen Entomologen, 54 : 30-38.

REDER G., 2004. - Neu- und Wiederfunde von Stechimmen im Naturraum Nördlicher Oberrheingraben von Rheinland-Pfalz und Hessen (Hymenoptera: Aculeata). Bembix, 18 : 26-32. 
— 2005. - Ergänzungen zur Hymenopterenfauna von Rheinland-Pfalz: Erste Nachweise von Miscophus eatoni S., Mimumesa beaumonti (V. Lith) (Sphecidae) und Chrysis sexdentata Chr. (Chrysididae) (Hymenoptera: Aculeata et Chalcidoidea). Fauna und Flora in Rheinland-Pfalz, 10 : 927-969.

RIEMANN H., 1995. - Zur Stechimmenfauna des Bremer Bürgerparks (Hymenoptera: Aculeata). Abhandlungen des naturwissenschaftlichen Vereins zu Bremen, 43 : 45-72.

— 1997. Die Stechimmenfauna der Weserdeiche bei Achim (Hym.: Aculeata). Drosera, 1997 : 45-64. 1999. - Weitere Nachweise und Betrachtungen zur Aculeatenfauna niedersächsischer Sandgruben (Hymenoptera: Aculeata). Abhandlungen des naturwissenschaftlichen Vereins zu Bremen, 44 : 825-846. SALZMANN-WANDELER I., 1998a. - Zur Insektenfauna der Umgebung der Vogelwarte Sempach, Kanton Luzern. XIX. Hymenoptera 5: Sphecidae (Grabwespen). Entomologische Berichte Luzern, 39 : 97-100.

— 1998b. Zur Insektenfauna von Gersau-Oberholz, Kanton Schwyz. XVI Hymenoptera 4 : Sphecidae (Grabwespen). Entomologische Berichte Luzern, 39 : 101-104.

SALZMANN-WANDELER I. \& REZBANYAI-RESER L., 1999. Zur Grabwespenfauna vom Monte Generoso, Kanton Tessin, Südschweiz (Hymenoptera: Sphecidae). Entomologische Berichte Luzern, 42 : 95-102.

— 2001. - Grabwespen aus der Umgebung von Meride und Lugano-Brè, Kanton Tessin, Südschweiz (Hymenoptera: Sphecidae). Entomologische Berichte Luzern, 45 : 151-160.

2004. - Grabwespen aus fünf Feuchtgebieten der Zentralschweiz (Hymenoptera: Sphecidae). Entomologische Berichte Luzern, 51 : 19-28.

SAURE C. \& DÜRRENFELD D., 1995. - Bienen und Wespen (Hymenoptera: Aculeata) der Gabower Hänge bei Bad Freienwalde (Kreis Märkisch-Oderland). Naturschutz und Landshaftspflegein Brandenburg, Heft $2: 23-32$.

SCHLÜTER C., 2002. - Bienen und Grabwespen anthropogener Standorte im Landkreis Ammerland (Hymenoptera: Aculeata). Drosera, 2002 : 133-158.

SCHMID-EGGER C., 1994. - Die faunistische Bedeutung alter Weinberge am Beispiel der Stechimmen (Hymenoptera, Aculeata) des Höllenberges bei Grünstadt. Fauna und Flora in Rheinland-Pfalz, $7: 673-707$.

1996. - Neue oder bemerkenswerte südwestdeutsche Stechimmenfunde. Bembix, 7 : 18-21.

2000. - Die Wildbienen- und Wespenfauna der oberrheinischen Trockenaue im südwestlichen Baden-Württemberg (Hymenoptera: Aculeata; Evanioidea). Vom Wildstrom zur Trockenaue Naturschutz - Spectrum - Themen, 92 : 257-306.

2001. - Die Stechimmerfauna des Bollenbergs in Südelsass (Hymenoptera, Aculeata). Bembix, $14: 9-22$.

— 2003. - New records of "Sphecidae" (Hymenoptera: Sphecidae \& Crabronidae) from Sicily (Italy) and Malta. Linzer biologische Beiträge, 35 : 747-762.

SCHMID-EGGER C. \& NIEHUIS O., 1997. - Ergänzungen und Berichtigungen zur Stechimmenfauna von Rheinland-Pfalz. Bembix, 8 : 13-16.

SCHMID-EGGER C., RISCH S. \& NIEHUIS O., 1995. - Die Wildbienen und Wespen in Rheinland-Pfalz (Hymenoptera, Aculeata). Verteilung, Ökologie und Gefährdungssituation. Fauna und Flora in Rheinland-Pfalz, Beih. 16 : 1-296.

SCHMIDT K., 1997. - Zur Variabilität und Verbreitung von Rhopalum beaumonti Moczar (Hymenoptera, Sphecidae). Bembix, $8: 43-45$.

SCHMIDT K. \& SCHMID-EGGER C., 1997. - Kritisches Verzeichnis der deutschen Grabwespenarten (Hymenoptera, Sphecidae). Mitteilungen der Arbeitsgemeinschaft ostwestfälisch-lippischer Entomologen, 13 (3) : 1-35.

SCHNEIDER N. \& CARRIÈRES E., 2004. - Capture de crabronides, sphécides et euménides au Bon-Pays (Luxembourg) à l'aide de pièges Malaise (Hymenoptera, Aculeata). Bulletin de la Société des Naturalistes luxembourgeois, 105 : 95-104.

SCHNEIDER N. \& WAHIS R., 1998. - Contribution à la connaissance des Vespiformes des carrières du Grès de Luxembourg et notes additionnelles sur quelques Pompilides (Hymenoptera, Aculeata). Archives - Institut grand-ducal de Luxembourg. Section des Sciences naturelles, physiques et mathématiques, n.s., 42 : 11-37.

SCHWARZ M., 1999. - Hautflügler (Hymenoptera) im Stadtgebiet von Linz. Naturkdliches Jahrbuch der Stadt Linz, 45 : 73-134. 
SKIBINSKA E., 1995. - Sphecidae (Aculeata) of subcontinental pine forest standes (Peucedano-Pinetum) of various ages in Puszcza Bialowieska. Fragmenta Faunistica, 38 : 419-433.

1999. - [State of knowledge of digger wasps (Sphecidae), social wasps (Vespidae), solitary wasps (Eumenidae), spider wasps (Pompilidae), and cuckoo wasps (Chrysididae) in the Bialowieza Forest. Parki Narodowe i Rezerwaty Przyrody, 18 : 93-104.

— 2001. - Superfamilia: Sphecoidea, p. 219-220. In J. M. Gutowski \& B. Jaroszewicz (eds.) [Catagogue of the fauna of Biolowieza Primeval Forest.] Instytut Badawczy Lesnictwa, Warszawa. 403 pp.

SMISSEN J. VAN DER, 1993. - Zweiter Beitrag zur Bienen- und Wespenfauna in südöstlichen SchleswigHolstein und nordöstlichen Niedersachsen (Hymenoptera: Aculeata). Drosera, 1993 : 125-134.

— 1998. - Beitrag zur Stechimmenfauna des mittleren und südlichen Schleswig-Holstein und angrenzender Begiete in Mecklenburg und Niedersachsen (Hymenoptera Aculeata). Mitteilungen der Arbeitsgemeinschaft ostwestfälisch-lippischer Entomologen, 14 (Beiheft 4) : 1-76.

SMIT J., 2000. - The wasps of Madeira (Hymenoptera: Chrysididae, Pompilidae, Vespidae, Sphecidae). Entomofauna, 21 : 165-180.

SÖRENSEN V., MAUSS V. \& SCHINDLER M., 1999. - Records of bees and wasps in the district Nordfriesland (Schleswig-Holstein) and surrounding areas (Hymenoptera Aculeata). Faunistisch-ökologische Mitteilungen, 7 : 497-508.

SORG M. \& WOLF H., 1993. - Naturkundliche Untersuchungen zum Naturschutzgebiet "Die Spey" (Stadt Krefeld, Kreis Neuss). II. Die Lebensgemeinschaften der Grabwespen (Hymenoptera, Aculeata, Sphecidae). Natur am Niederrhein (N. F.), 8 : 58-72.

SRBA M., 1999. - [Sphecid wasps (Hymenoptera, Sphecoidea) of family Crabronidae living in surroundings of Podkrusnohorsky Zoopark Chomutov]. Fauna Bohemiae Septentionalis, 24 : 179-182 [en tchèque].

STANDFUSS K. \& STANDFUSS L., 2006a. - Hautflügler eines Grossstadtgardens nach zwanzigjähriger Florenförderung (Insecta: Hymenoptera). Entomofauna, 27 : 81-92.

— 2006b. - Zum aktuellen Artenbestand der Astatinae, Larrinae, Crabroninae und Philanthinae (Hymenoptera: Crabronidae p.p.) der planar-kollinen Vegetationsstufe in Südost-Thessalien/Griechland. Entomofauna, 27: 93-104.

Stolle E., Burger F. \& Drewes B., 2004. - Rote Liste der Grabwespen (Hymenoptera: "Sphecidae") des Landes Sachsen-Anhalt. Berichte des Landesamtes für Umweltschutz Sachsen-Anhalt, 39 : 369-375.

STOYANOV I., 1996. - Notizen über die Biologie von Ectemnius confinis (Walker, 1871) (Hymenoptera: Sphecidae), eine neue Grabwespenart für die Fauna Bulgariens. Bembix, 6 : 15-16.

StOYANOV I. \& LJUBOMIROV T., 1997. - A preliminary faunistic list and zoogeographical analysis of the gold and digger wasps (Hymenoptera: Chrysididae, Sphecidae) from the Rila Mountain. Acta Entomologica Bulgarica, 3 : 24-29.

SZCZEPKO K. \& KOWALCZYK J.K., 2001. - Sphecid wasps (Hymenoptera: Sphecidae) in habitats of abandoned village in forest territory in Kampinoski National Park (Poland). Polskie Pismo Entomologiczne, 70 : 185-193.

— 2004. - Post-agriculture habitats as a refuge area for sphecid wasps (Hymenoptera: Sphecidae) in the forest territory of the Kampinos National Park. Ecological Questions, 4 : 123-132.

THEUNERT R., 1994a. - Neue Fundorte für einige nach dem zweiten Weltkrieg nur spärlich bekanntgewordene Stechimmen Niedersachsens (Hymenoptera). Entomologische Nachrichten und Berichte, 38 : 276-279.

1994b. - Kommentiertes Verzeichnis der Stechimmen Niedersachsens und Bremens (Insecta: Hymenoptera Aculeata). Oekologieconsult-Schrift, 1 : 1-112.

1999. - Neue Fundorte für einige nach dem zweiten Weltkrieg nur spärlich bekannt gewordene Stechimmen Niedersachsens (Hym.), Folge III. Entomologische Nachrichten und Berichte, 43 : 137-139. 2005a. - Verzeichnis der Stechimmen Niedersachsens und Bremens (Hymenoptera Aculeata). Bembix, 20 : 10-26.

2005b. - Hervorhebenswerte Stechimmenfunde aus dem östlichen Niedersachsen (Hymenoptera),

Folge III. Beiträge zur Naturkunde Niedersachsens, 58 : 7-9.

- 2007a. - Zur Lebensweise von Crossocerus congener (Dahlbom, 1844) (Hymenoptera: Sphecidae). Bembix, 24 : 30-32. 
2007b. - Zum Sexualverhalten der Grabwespe Ectemnius lapidarius (Panzer, 1804) (Hymenoptera: Sphecidae). Bembix, 25 : 22-26.

2007c. - Hervorhebenswerte Stechimmenfunde aus dem östlichen Niedersachsen (Hymenoptera), Folge IV. Beiträge zur Naturkunde Niedersachsens, 60 : 95-99.

2008. - Atlas zur Verbreitung der Grabwespen (Hym.: Sphecidae s.1.) in Niedersachsen und Bremen (1978-2007). Oekologieconsult-Schrift, 6, 98 pp.

TISCHENDORF S., 2001. - Wildbienen und Wespen (Hymenoptera: Aculeata) in Niedersachsen und Bremen "Kükopf-Knoblauchsaue" (Hessen). Hessische faunitische Briefe, 20 : 21-42.

TISCHENDORF S. \& FROMER U., 2004. - Stechimmen (Hymenoptera: Aculeata) an xerothermen Hanglagen in oberen Mittelrheinteil bei Lorch unter Berücksichtigung ihrer Verbreitung in Naturraum und in Hessen. Hessische faunitische Briefe, 23 : 25-122.

TiSCHENDORF S. \& TREIBER R., 2003. - Stechimmen (Hymenoptera, Aculeata) under Hochspannungsfreiteilungen im Rhein-Mainz-Gebiet. Carolinea. Beiträge zur naturkundlichen Forschung in Südwestdeutschland, 60 : 113-130.

TOMARChIO S. \& TURRISI G. F., 2006. - New or little known Sphecidae (Hymenoptera, Aculeata) from Sicily (Italy). Linzer biologische Beiträge, 38 : 953-969.

TORMOS J., ASIS J. D. \& GAYUBO S. F., 1994. - Esfecidofauna de la provincia de Albacete (Hymenoptera: Sphecidae). Revista de Estudios albacetenses, 20 (34) : 183-246.

TORMOS J., Asis J. D., GAYUBO S. F., PORTILlo M. \& TORRES F., 2000. - Nesting behavior of Oxybelus lamellatus Olivier (Hymenoptera: Sphecidae). Annals of the Entomological Society of America, 93 : 326-332.

Torregrosa S. G., GAyUbo S. F., Tormos J. \& Asis J. D., 1993. - Esfecidos de la provincia de Alicante (Hymenoptera: Sphecidae). Boletin de la Asociacion española de Entomologia, 17 : 9-25.

UFFEN R.W., 1996. - Rhopalum coarctatum (Scop.) (Hymenoptera: Sphecidae) nesting in a case of Taleporia tubulosa Retz. (Lepidoptera: Psychidae). British Journal of Entomology and Natural History, 9 : 108.

VAGO J.-L., 2005a. - Le genre Crossocerus Lepeletier \& Brullé dans le Nord de la France (Hymenoptera, Sphecidae, Crabroninae). Bulletin de la Société entomologique du Nord de la France, 314 : 12-21. 2005b. - Deux Sphecidae Crabroninae peu communs observés dans l'Aisne : Ectemnius nigritarsus (Herrich-Schaeffer) et Lindenius subaeneus Lepeletier \& Brullé (Hymenoptera). Bulletin de la Société entomologique du Nord de la France, 316 : 19.

VAN DER MEER, 2002. [The aculeates of Meijendel]. Entomologische Berichten, 21 : 14-16.

VEPREK D. \& STRAKA J., 2007. - Apoidea Spheciformes (Kutilky). In Bogusch, Straka \& Kment (eds.). Annotated checklist of the Aculeata (Hymenoptera) of the Czech Republik and Slovakia. Acta entomologica Musei nationalis Pragae, Suppl. 11 [Crabronidae : p. 118-232].

VERNIER R. 1994. - Deux espèces rares du genre Crabro F. (Hymenoptera, Sphecidae) nichent encore sur le Plateau Suisse. Bulletin romand d'Entomologie, 12 : 95-100.

VIKBERG V., 1999. - Crossocerus assimilis and Bethylus boops, two aculeate wasps new to the fauna of Finland (Hymenoptera, Crabronidae and Bethylidae). Entomologica Fennica, 10 : 245-246.

Voblenko A. S., Gorobchishin V. A. \& Nesterov M. A., 1996. - Digger wasps (Hymenoptera, Sphecidae) of Ukrainian Polesye. Sphecos, 30 : 14-15.

WEBER K. 1998. - Revision der "Wespensammlung" von T. Schneid in Naturkundemuseum Bamberg (Hymenoptera: "Scolioidea", Pompilidae, Vespidae und Sphecidae). Bericht der naturforschende Gesellschaft in Bamberg, 72 : 113-156.

WICKL A. \& WICKL K.-H., 1994. - Seltene und bemerkenswerte Bienen und Wespen aus der Oberpfalz (Hymenoptera Aculeata). Acta Albertina Ratisbonensia, 49 : 189-198.

WICKL K.-H., 1999. - Bemerkenswerte Vorkommen von Bienen, Wespen und Ameisen in Sandgebieten der Oberpfalz (Hymenoptera Aculeata). Galathea, 15 : 95-119.

WiSNIOWSKI B., 1993. - [Materials on sphecid-wasps (Hymenoptera, Sphecidae) of the Bialowieza Forest]. Parki Narodowe i Rezerwaty Przyrody, 12 : 93-99.

- 2000. - [Hymenoptera of the Polish Bieszczady Mts with the special regard to the Bieszczady National Park]. Monografie Bieszczadzkie, 8 : 145-187.

2002. - [Tiphiidae, Sapygidae, Mutillidae, Pompilidae, Eumenidae, Vespidae and Sphecidae (Hymenoptera: Aculeata) of Ojców National Park. Part I. List of species and zoogeographical analysis]. Parki Narodowe i Rezerwaty Przyrody, 21 : 51-82. 
— 2004. - Annotated list of Polish digger wasps (Hymenoptera: Sphecidae). Polskie Pismo Entomologiczne, $73: 33-63$.

— 2005a. - [Tiphiidae, Sapygidae, Mutillidae, Pompilidae, Eumenidae, Vespidae and Sphecidae (Hymenoptera: Aculeata) of Ojców National Park. Part 2. Analysis of aculeate assemblages]. Pradnik Prace i materiali Muzeum im. Prof. Wladyslava Szafera, 15 : 311-338.

— 2005b. - [Tiphiidae, Sapygidae, Mutillidae, Pompilidae, Eumenidae, Vespidae and Sphecidae (Hymenoptera: Aculeata) of Ojców National Park. Part 3. Evaluation and state of knowledge im Poland]. Pradnik Prace i materiali Muzeum im. Prof. Wladyslava Szafera, 15 : 339-356.

WiSNIOWSKI B. \& KOWALCZYK J. K., 1998. - [Some digger wasps new to Polish fauna with notes on two other species (Hymenoptera: Aculeata: Sphecidae)]. Pradnik Prace i materiali Muzeum im. Prof. Wladyslava Szafera, 11-12 : 219-222.

WISNIOWSKI B. \& WERSTAK K., 2003. [Contribution to the knowledge of digger wasps (Hymenoptera: Sphecidae) of Pieniny National Park]. Wiadomosci Entomologiczne, 22 : 73-80.

WITT R., 1996. - Beitrag zur Grabwespenfauna Brandenburgs (Hymenoptera: Sphecidae). Drosera, 1996 : 103-112.

WOLF H., 1993. - Beitrag zur Stechimmen-Fauna (Hymenoptera, Aculeata) einer Brenne bei Gersthoffen. Berichte des naturwissenschaftlichen Vereins für Schwaben, 97 : 13-15.

— 1995. - Stechimmen an den Rheinhängen nahe der Loreley (Hymenoptera: Aculeata). Miteilungen des internationalen entomologischen Vereins, 20 : 15-21.

— 2001. - Stechimmen (Hymenoptera aculeata) des Lechtals von Augsburg bis zur Lechmündung. Berichte des naturwissenschaftlichen Vereins für Schwaben, 2001 : 168-185.

— 2003. - Die Stechimmen (Hymenoptera Aculeata) des Naturschutzgebietes Bommeckeltal in Plettenberg (Sauerland). Sauerländische Naturbeobachter, 28 : 256-268.

WOYDAK H., 1996. - Hymenoptera Aculeata Westfalica. Familia: Sphecidae (Grabwespen). Abhandlungen aus dem Westfälischen Museum für Naturkunde, 58 : 1-135.

ZEHNDER G. \& ZETTEL J., 1999. - Auensukzession und Zonation im Rottensand (Pfynwald, Kt.VS). I. Wiederbesiedlung einer Überschwemmungsfläche durch Grabenwespen (Hymenoptera, Sphecidae). Mitteilungen der schweizerischen entomologischen Gesellschaft, 72 : 123-137.

ZETTEL H., 2000. - Seltene und bemerkenswerte Grabwespen (Hymenoptera: Spheciformes) aus Ostösterreich. Beiträge zur Entomofaunistik, 1 : 19-33.

— 2004. - Weitere Notizen zu einigen Grabwespen im Osten Oesterreichs (Hymenoptera: Sphecidae, Pemphredonidae, Crabronidae, Nyssonidae). Beiträge zur Entomofaunistik, 5 : 3-8.

ZeTTEL H., GROSS H. \& MAZZUCCO K., 2001. Liste der Grabwespen-Arten (Hymenoptera: Spheciformes) Wiens, Österreich. Beiträge zur Entomofaunistik, 2 : 61-86.

Zettel H., LJUbOMirov T., Steiner F. M., SCHLiCK-Steiner B. C., GRABENWEGER G. \& WiESBAUER H., 2004. - The european ant hunters Tracheliodes curvitarsis and T. varus (Hymenoptera: Crabronidae): taxonomy, species discrimination, distribution, and biology. Myrmecologische Nachrichten, $6: 39-47$.

ZETTEl H., WiESBAUER H. \& ZiMMERMANN D., 2008. - Weitere interessante Grabwespenvorkommen (Hymenoptera: Sphecidae, Crabronidae) in Osten Oesterreichs. Beiträge zur Entomofaunistik, 8 : 133-140.

ZISKA T., 2002. - Untersuchungen zur Vorkommen von aculeaten Hymenopteren im Feuchtgebiet Krumme Lake. Märkische entomologische Nachrichten, 4 : 1-16.

\section{ANNEXE}

Classification simplifiée des Apoidea, d'après PrENTICE (1998). Seuls les noms des taxa présents en Europe ont été retenus. Les genres sont classés par ordre alphabétique au sein de chaque tribu ou sous-tribu.

Famille Heterogynaidae Nagy : Heterogyna.

Famille Ampulicidae Shuckard

Tribu Dolichurini Dahlbom : Dolichurus.

Tribu Ampulicini Shuckard : Ampulex. 
Famille Sphecidae Latreille, nouveau rang

Tribu Sceliphrini Ashmead : Chalybion, Sceliphron.

Tribu Sphecini Latreille

Sous-tribu Sphecina Latreille : Isodontia, Sphex.

Sous-tribu Prionychina Bohart \& Menke : Chilosphex, Palmodes, Prionyx.

Tribu Ammophilini André : Ammophila, Eremochares, Hoplammophila, Podalonia.

Famille Crabronidae Latreille, nouveau rang

Sous-famille Astatinae Lepeletier

Tribu Astatini Lepeletier : Astata, Dryudella.

Sous-famille Bembicinae Latreille

Tribu Alyssonini Dalla Torre : Alysson, Didineis.

Tribu Nyssonini Latreille : Brachystegus, Nysson, Synnevrus.

Tribu Bembicini Latreille

Sous-tribu Clitemnestrina Nemkov \& Lelej, sensu novo : Olgia.

Sous-tribu Gorytina Lepeletier : Argogorytes, Gorytes, Harpactus, Hoplisoides, Lestiphorus, Oryttus, Psammaecius, Pseudoplisus.

Sous-tribu Handlirschiina Nemkov \& Lelej : Ammatomus, Sphecius.

Sous-tribu Stizina Costa, sensu novo : Stizoides, Stizus.

Sous-tribu Bembecinina Prentice : Bembecinus.

Sous-tribu Bembicina Latreille : Bembix.

Sous-famille Philanthinae Latreille

Tribu Philanthini Latreille, sensu novo

Sous-tribu Philanthina Latreille : Philanthus.

Tribu Pseudoscoliini Menke : Pseudoscolia.

Tribu Cercerini Lepeletier : Cerceris.

Sous-famille Pemphredoninae Dahlbom

Tribu Entomosericini Dalla Torre, sensu novo : Entomosericus.

Tribu Psenini Costa : Mimesa, Mimumesa, Psen, Pseneo, Psenulus.

Tribu Pemphredonini Dahlbom

Sous-tribu Pemphredonina Dahlbom : Diodontus, Passaleocus, Pemphredon, Polemistus.

Sous-tribu Spilomenina Menke : Spilomena.

Sous-tribu Stigmina Bohart \& Menke : Carinostigmus, Stigmus.

Sous-tribu Ammoplanina Bohart \& Menke : Ammoplanellus, Ammoplanus.

Sous-famille Mellininae Latreille

Tribu Mellinini Latreille : Mellinus.

Sous-famille Dinetinae Fox, nouveau rang

Tribu Dinetini Fox : Dinetus.

Sous-famille Crabroninae Latreille

Tribu Palarini Börner, sensu novo : Palarus.

Tribu Larrini Latreille

Sous-tribu Larrina Latreille : Larra, Liris.

Sous-tribu Gastrosericina André : Ancistromma, Gastrosericus, Holotachysphex, Prosopigastra, Tachysphex, Tachytes.

Tribu Miscophini Fox : Miscophus, Nitela, Plenoculus, Solierella.

Tribu Trypoxylini Lepeletier : Pison, Trypoxylon.

Tribu Oxybelini Leach : Belomicroides, Belomicrus, Oxybelus.

Tribu Crabronini Latreille

Sous-tribu Anacrabronina Ashmead, sensu novo : Encopognathus, Entomognathus.

Sous-tribu Crabronina Latreille : Crabro, Crossocerus, Ectemnius, Lestica, Lindenius, Rhopalum, Tracheliodes.

Famille Apidae Latreille 